quatrième série-tome $46 \quad$ fascicule 3 mai-juin 2013

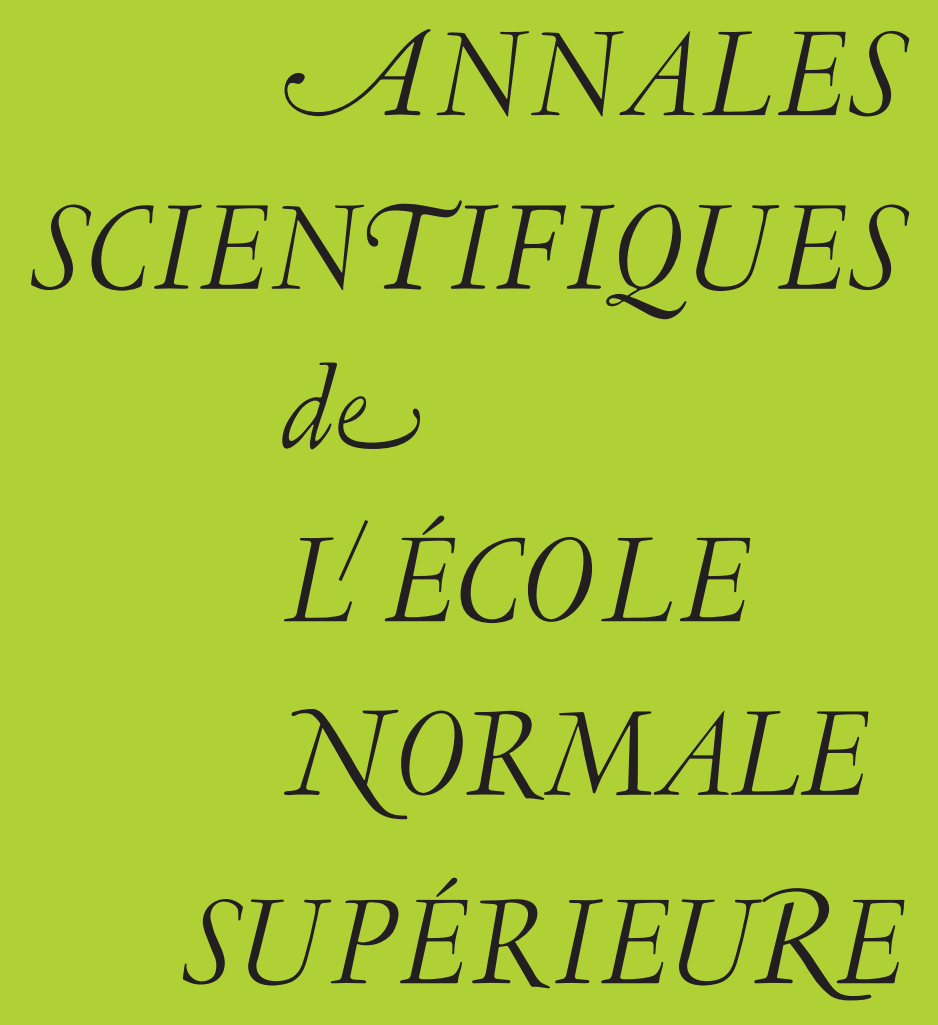

\title{
Claire VOISIN
}

The generalized Hodge and Bloch conjectures are equivalent for general complete intersections 
Ann. Scient. Éc. Norm. Sup.

$4^{\text {e }}$ série, t. 46, 2013, p. 449 à 475

\title{
THE GENERALIZED HODGE AND BLOCH CONJECTURES ARE EQUIVALENT FOR GENERAL COMPLETE INTERSECTIONS
}

\author{
BY Claire VOISIN
}

\begin{abstract}
We prove that Bloch's conjecture is true for surfaces with $p_{g}=0$ obtained as 0 -sets $X_{\sigma}$ of a section $\sigma$ of a very ample vector bundle on a variety $X$ with "trivial" Chow groups. We get a similar result in presence of a finite group action, showing that if a projector of the group acts as 0 on holomorphic 2 -forms of $X_{\sigma}$, then it acts as 0 on 0 -cycles of degree 0 of $X_{\sigma}$. In higher dimension, we also prove a similar but conditional result showing that the generalized Hodge conjecture for general $X_{\sigma}$ implies the generalized Bloch conjecture for any smooth $X_{\sigma}$, assuming the Lefschetz standard conjecture (the last hypothesis is not needed in dimension 3 ).

RÉsumÉ. - Nous montrons la conjecture de Bloch pour les surfaces avec $p_{g}=0$ obtenues comme lieux des zéros $X_{\sigma}$ d'une section $\sigma$ d'un fibré vectoriel très ample sur une variété $X$ à groupes de Chow «triviaux ». Nous obtenons un résultat similaire en présence d'une action d'un groupe fini, montrant que si un projecteur du groupe agit comme 0 sur les 2-formes holomorphes de $X_{\sigma}$, il agit comme 0 sur les 0 -cycles de degré 0 de $X_{\sigma}$. En dimension supérieure, nous obtenons un résultat similaire mais conditionnel montrant que la conjecture de Hodge généralisée pour $X_{\sigma}$ générale entraine la conjecture de Bloch généralisée pour tout $X_{\sigma}$ lisse, en supposant satisfaite la conjecture de Lefschetz standard (cette dernière hypothèse n'étant pas nécessaire en dimension 3 ).
\end{abstract}

\section{Introduction}

Recall first that a weight $k$ Hodge structure $\left(L, L^{p, q}\right)$ has Hodge coniveau $c \leq \frac{k}{2}$ if the Hodge decomposition of $L_{\mathbb{C}}$ takes the form

$$
L_{\mathbb{C}}=L^{k-c, c} \oplus L^{k-c-1, c+1} \oplus \cdots \oplus L^{c, k-c}
$$

with $L^{k-c, c} \neq 0$. If $X$ is a smooth complex projective variety and $Y \subset X$ is a closed algebraic subset of codimension $c$, then $\operatorname{Ker}\left(H^{k}(X, \mathbb{Q}) \rightarrow H^{k}(X \backslash Y, \mathbb{Q})\right)$ is a sub-Hodge structure of coniveau $\geq c$ of $H^{k}(X, \mathbb{Q})$ (cf. [34, Theorem 7]). The generalized Hodge conjecture formulated by Grothendieck [10] is the following. 
Conjecture 1.1. - Let $X$ be as above and let $L \subset H^{k}(X, \mathbb{Q})$ be a sub-Hodge structure of Hodge coniveau $\geq c$. Then $L$ has geometric coniveau $\geq c$, i.e., there exists a closed algebraic subset $Y \subset X$ of codimension $c$ such that

$$
L \subset \operatorname{Ker}\left(H^{k}(X, \mathbb{Q}) \rightarrow H^{k}(X \backslash Y, \mathbb{Q})\right) .
$$

This conjecture is widely open, even for general hypersurfaces or complete intersections in projective space (cf. [33]). Consider a smooth complete intersection $X \subset \mathbb{P}^{n}$ of $r$ hypersurfaces of degrees $d_{1} \leq \cdots \leq d_{r}$. Then the Hodge coniveau of the Hodge structure on $H^{n-r}(X, \mathbb{Q})_{\text {prim }}$ (the only part of the cohomology of $X$ which does not come from the ambient space) is given by the formula (cf. [33], where complete intersections of coniveau 2 are studied):

$$
\operatorname{coniveau}\left(H^{n-r}(X, \mathbb{Q})_{\text {prim }}\right) \geq c \Leftrightarrow n \geq \sum_{i} d_{i}+(c-1) d_{r} .
$$

A deep relation between Chow groups and geometric coniveau appears in the various generalizations of Mumford's theorem obtained in [3], [26], [16], [14], [22], based on refinements of the diagonal decomposition principle due to Bloch and Srinivas. The resulting statement is the following (see [32, II,10.3.2]):

Theorem 1.2. - Let $X$ be a smooth projective variety of dimension $m$. Assume that the cycle class map

$$
\mathrm{cl}: C H_{i}(X)_{\mathbb{Q}} \rightarrow H^{2 m-2 i}(X, \mathbb{Q})
$$

is injective for $i \leq c-1$. Then for any $k \geq 0$, the geometric coniveau of the Hodge structure $H^{k}(X, \mathbb{Q})^{\perp \text { alg }}$ is greater than or equal to $c$.

Here $H^{k}(X, \mathbb{Q})^{\perp \text { alg }}$ denotes the "transcendental part" of the cohomology, that is, the set of classes which are orthogonal to all cycle classes via Poincare duality. It is of course different from $H^{k}(X, \mathbb{Q})$ only if $k$ is even.

This theorem could be a possible way to attack Conjecture 1.1 for the whole cohomology of $X$, or at least its transcendental part. The first case of this theorem, that is the case where $c=1$, was obtained by Bloch-Srinivas [3]. It says that if a variety $X$ has $C H_{0}(X)=\mathbb{Z}$, then $H^{k, 0}(X)=0$ for any $k>0$ (which generalizes Mumford's theorem [17]) and furthermore, the cohomology of positive degree of $X$ is supported on a proper algebraic subset $Y \subset X$ (which solves Conjecture 1.1 for such $X$ and for coniveau 1).

The next major open problem, which by the above theorem would solve the generalized Hodge conjecture, is the following conjecture relating the Hodge coniveau and Chow groups. This conjecture is a vast generalization of Bloch conjecture for surfaces [2].

Conjecture 1.3 (cf. [32, II, 11.2.2]). - Let $X$ be a smooth projective variety of dimension $m$ satisfying the condition $H^{p, q}(X)=0$ for $p \neq q$ and $p<c($ or $q<c)$. Then for any integer $i \leq c-1$, the cycle class map

$$
\mathrm{cl}: C H_{i}(X)_{\mathbb{Q}} \rightarrow H^{2 m-2 i}(X, \mathbb{Q})
$$

is injective.

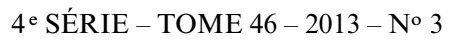


Note that assuming the Hodge conjecture, the assumptions are equivalent to saying that the cohomology $H^{*}(X, \mathbb{Q})^{\perp \text { alg }}$ has Hodge coniveau $\geq c$.

If we look at the case of hypersurfaces or complete intersections in projective space, we see from (1) that Conjecture 1.3 predicts the following:

Conjecture 1.4. - Let $X \subset \mathbb{P}^{n}$ be a smooth complete intersection of hypersurfaces of degrees $d_{1} \leq \cdots \leq d_{r}$. Then if $n \geq \sum_{i} d_{i}+(c-1) d_{r}$, the cycle class map

$$
\mathrm{cl}: C H_{i}(X)_{\mathbb{Q}} \rightarrow H^{2 n-2 r-2 i}(X, \mathbb{Q})
$$

is injective for any integer $i \leq c-1$.

Note that, by Theorem 1.2, this conjecture would imply Conjecture 1.1 for very general complete intersections, that is, Conjecture 1.5 below. Indeed, by monodromy arguments, the Hodge structure on the primitive middle cohomology of a very general complete intersection is simple except for some rare and classified cases where it is made of Hodge classes. Thus in this case a nontrivial sub-Hodge structure must be the whole primitive cohomology, and its Hodge coniveau is computed by (1).

Apart from very particular values of the degrees $d_{i}$ (like complete intersections of quadrics [21], or cubics of small dimension [1]), Conjecture 1.4 is essentially known only in the case $c=1$, where the considered complete intersections are Fano, hence rationally connected, so that the equality $C H_{0}(X)=\mathbb{Z}$ is trivial in this case.

In the paper [31], it is proved that for any pair $(n, d)$, there are smooth hypersurfaces of degree $d$ in $\mathbb{P}^{n}$ satisfying the conclusion of Conjecture 1.4.

Coming back to Conjecture 1.1 for general complete intersections in projective space, we get from (1) that it is equivalent to the following statement:

Conjecture 1.5. - The primitive cohomology $H^{n-r}(X, \mathbb{Q})_{\text {prim }}$ of a smooth complete intersection $X \subset \mathbb{P}^{n}$ of $r$ hypersurfaces of degrees $d_{1} \leq \cdots \leq d_{r}$ vanishes on the complement of a closed algebraic subset $Y \subset X$ of codimension $c$ if $n \geq \sum_{i} d_{i}+(c-1) d_{r}$.

As already mentioned, Conjecture 1.5 would be implied by Conjecture 1.4 using Theorem 1.2. The paper [33] is an attempt to prove directly Conjecture 1.5 for hypersurfaces or complete intersections of coniveau 2 without trying to show the triviality of their $\mathrm{CH}_{0}$ and $\mathrm{CH}_{1}$ groups. Conjecture 1.5 is shown there to be implied by a conjecture on the effective cone of algebraic cycles (on some auxiliary variety). This work was motivated by the fact that, apart from the case of coniveau 1, as is apparent from the lack of progress in this direction and the fact that the results obtained this way (see [7], [22], [20]) cover a numerical range which is rather different from the one predicted by Conjecture 1.5, it seems now unlikely that one will prove Conjecture 1.5 by an application of Theorem 1.2, that is via the proof of the triviality of Chow groups of small dimension.

In fact, we will essentially show in this paper that for very general complete intersections of ample hypersurfaces or more generally, zero sets of sections of very ample vector bundles inside any smooth projective variety $X$ with "trivial" Chow groups, Conjecture 1.1 (that is Conjecture 1.5 if the ambient space is $\mathbb{P}^{n}$ ) implies Conjecture 1.3 (that is Conjecture 1.4 if the ambient space is $\mathbb{P}^{n}$ ). Stated this way, this is not completely correct, and we have to add an extra assumption that we now explain. 
Let us state the following conjecture, that we will relate later on (cf. Proposition 2.6) to the so-called standard conjectures [13]:

Conjecture 1.6. - Let $X$ be a smooth complex projective variety, and let $Y \subset X$ be a closed algebraic subset. Let $Z \subset X$ be a codimension $k$ algebraic cycle, and assume that the cohomology class $[Z] \in H^{2 k}(X, \mathbb{Q})$ vanishes in $H^{2 k}(X \backslash Y, \mathbb{Q})$. Then there exists a codimension $k$ cycle $Z^{\prime}$ on $X$ with $\mathbb{Q}$-coefficients, which is supported on $Y$ and such that $\left[Z^{\prime}\right]=[Z]$ in $H^{2 k}(X, \mathbb{Q})$.

Our main result in this paper is the following theorem. We will say here that a smooth variety $X$ has trivial Chow groups if the cycle map cl $: C H^{i}(X)_{\mathbb{Q}} \rightarrow H^{2 i}(X, \mathbb{Q})$ is injective for any $i$. In the theorem below, we say that a vector bundle $E$ on $X$ is very ample if for any subscheme $z \subset X$ of length 2 , the restriction map $H^{0}(X, E) \rightarrow H^{0}\left(z, E_{\mid z}\right)$ is surjective.

TheOREm 1.7. - Assume Conjecture 1.6 holds for cycles of codimension $n-r$. Let X be a smooth complex projective n-fold with trivial Chow groups. Let $E$ be a very ample vector bundle on $X$ of rank $r$. Assume that for a very general subvariety $X_{b} \subset X$ defined as the zero locus of a section of $E$, the Hodge structure on $H^{n-r}\left(X_{b}, \mathbb{Q}\right)_{\text {van }}$ is supported on a closed algebraic subset $Y_{b} \subset X_{b}$ of codimension $\geq c$. Then for the general such $X_{b}$ (hence in fact for all), the cycle map cl : $C H_{i}\left(X_{b}\right)_{\mathbb{Q}} \rightarrow H^{2 n-2 r-2 i}\left(X_{b}, \mathbb{Q}\right)$ is injective for any $i<c$.

Here the space $H^{n-r}\left(X_{b}, \mathbb{Q}\right)_{\text {van }}$ of vanishing cohomology is defined as

$$
\operatorname{Ker}\left(j_{b *}: H^{n-r}\left(X_{b}, \mathbb{Q}\right) \rightarrow H^{n+r}(X, \mathbb{Q})\right),
$$

where $j_{b}$ is the inclusion of $X_{b}$ in $X$. If the ambient space $X$ is $\mathbb{P}^{n}$, the vanishing cohomology is nothing but the primitive cohomology with respect to the line bundle $\theta_{X_{b}}(1)$.

As a particular case, we get:

Corollary 1.8. - Assuming Conjecture 1.6 for cycles of codimension $n-r$, the generalized Hodge Conjecture 1.5 implies the generalized Bloch Conjecture 1.4 for complete intersections in projective space.

These results are conditional results. However, in small dimension, some assumptions are automatically satisfied, and this gives us the following statement, which will be proved in Subsection 3.3.

Theorem 1.9. - Let $X$ be a smooth complex projective variety of dimension $n$ with trivial Chow groups. Let $E$ be a very ample vector bundle of rank $r$ on $X$. Then we have:

1) Case $n-r=2$. If the smooth surfaces $X_{b}$ obtained as zero sets of sections of $E$ have $h^{2,0}\left(X_{b}\right)=0$, then they satisfy $C H_{0}\left(X_{b}\right)=\mathbb{Z}$. (This is the Bloch conjecture).

2) Case $n-r=3$. If very general threefolds $X_{b}$ obtained as zero sets of sections of $E$ have the property that the degree 3 cohomology of $X_{b}$ is of geometric coniveau 1 (which is also equivalent to the fact that Abel-Jacobi map $C H^{2}\left(X_{b}\right)_{\mathrm{hom}} \rightarrow J^{3}\left(X_{b}\right)$ is surjective), then the Chow group $C H_{0}\left(X_{b}\right)$ is equal to $\mathbb{Z}$.

4 e SÉRIE - TOME $46-2013$ - No 3 
The proof of Theorem 1.7 can be also applied to get results for many complete intersections or zero sets of sections of very ample vector bundles $X_{b}$ endowed with the action of a finite group $G$. In this case, the method applies as well to the $\chi$-invariant part of $C H\left(X_{b}\right)$ where $\chi: G \rightarrow\{1,-1\}$ is a character, and more generally to any projector $\pi \in \mathbb{Q}[G]$.

REMARK 1.10. - As already mentioned, our results are unconditional in the case of surfaces or threefolds, where the needed assumptions will be satisfied by the Lefschetz theorem on $(1,1)$-classes. This is uninteresting in the case of complete intersections surfaces or threefolds in projective space, since those of coniveau 1 are Fano, but in the presence of a group action, there are interesting non trivial examples of group actions on complete intersection surfaces where this applies, in particular the Godeaux surfaces considered in [29], or the three-dimensional examples with group actions considered in [29] or in [23].

Other potential applications concern self-products of Calabi-Yau hypersurfaces. It was noticed in [30] that the generalized Bloch conjecture implies the following: Let $X$ be a $n$-dimensional smooth projective variety with $H^{i}\left(X, \theta_{X}\right)=0$ for $0<i<n$ and $H^{n}\left(X, \theta_{X}\right)=\mathbb{C}$ (for example $X$ could be a Calabi-Yau manifold). Then if $n$ is even the antisymmetric product $z \times z^{\prime}-z^{\prime} \times z$ of two 0 -cycles of $X$ of degree 0 should be 0 in $C H_{0}(X \times X)$. If $n$ is odd the symmetric product $z \times z^{\prime}+z^{\prime} \times z$ of two 0 -cycles of $X$ of degree 0 should be 0 in $C H_{0}(X \times X)$. This comes from Conjecture 1.3, or rather its generalization to motives, and from the observation that the Hodge structure on $\bigwedge^{2} H^{n}(X, \mathbb{Q})$ has Hodge coniveau $\geq 1$ (see Lemma 4.6).

We will show in Section 4:

Theorem 1.11. - Assume Conjecture 1.6. Let X be a very general Calabi-Yau hypersurface in projective space $\mathbb{P}^{n}$. Then if the generalized Hodge conjecture is true for the coniveau 1 Hodge structure on $\bigwedge^{2} H^{n-1}(X, \mathbb{Q})$ ( seen as a sub-Hodge structure of $H^{2 n-2}(X \times X, \mathbb{Q})$ ), the antisymmetric product $z \times z^{\prime}-z^{\prime} \times z$ of two 0 -cycles of $X$ of degree 0 is equal to 0 in $C H_{0}(X \times X)$ for $n-1$ even and the symmetric product $z \times z^{\prime}+z^{\prime} \times z$ of two 0 -cycles of $X$ of degree 0 is equal to 0 in $C H_{0}(X \times X)$ for $n-1$ odd.

The paper is organized as follows: in Section 2, we will show that Conjecture 1.6 is implied by the so-called Lefschetz standard conjecture. In Section 3, we will prove Theorem 1.7 and Theorem 1.9. Section 4 will provide a number of other geometric applications. For example, we will show how to recover the results of [29], or [23].

\section{Thanks}

I thank Manfred Lehn, Christoph Sorger and Burt Totaro for their help concerning Lemma 4.12, and especially Manfred Lehn and Christoph Sorger for communicating the text [15]. I thank the referee for useful suggestions, and in particular for an elegant argument simplifying the proof of Proposition 2.6. 


\section{Remarks on Conjecture 1.6}

The aim of this section is to comment on Conjecture 1.6. The first observation to make is the following:

Lemma 2.1. - Conjecture 1.6 is satisfied by codimension $k$ cycles $Z$ on $X$ whose cohomology class vanishes away from a codimension $k-1$ closed algebraic subset $Y$.

In particular, Conjecture 1.6 is satisfied by codimension 2 cycles.

Proof. - Indeed, if we have a codimension $k$ cycle $Z \subset X$, whose cohomology class $[Z] \in H^{2 k}(X, \mathbb{Q})$ vanishes on the open set $X \backslash Y$, where codim $\mathrm{Y} \geq k-1$, then we know (cf. [34, Proposition 3]) that there are Hodge classes $\alpha_{i} \in H d g^{2 k-2 c_{i}}\left(\widetilde{Y}_{i}, \mathbb{Q}\right)$, such that

$$
[Z]=\sum_{i} \tilde{j}_{i *} \alpha_{i}
$$

where $\tilde{j}_{i}: \widetilde{Y}_{i} \rightarrow X$ are desingularizations of the irreducible components $Y_{i}$ of $Y$, and $c_{i}:=\operatorname{codim} Y_{i}$.

As $c_{i} \geq k-1$ for all $i$ 's, the classes $\alpha_{i}$ are cycle classes on $\widetilde{Y}_{i}$ by the Lefschetz theorem on $(1,1)$-classes, which concludes the proof.

We are now going to relate precisely Conjecture 1.6 to the famous "standard conjectures" [13]. Let $X$ be a smooth projective variety of dimension $n$. The Künneth decomposition of $H^{*}(X \times X, \mathbb{Q})$ gives:

$$
H^{m}(X \times X, \mathbb{Q}) \cong \bigoplus_{p+q=m} H^{p}(X, \mathbb{Q}) \otimes H^{q}(X, \mathbb{Q}) .
$$

Poincaré duality on $X$ allows to rewrite this as

$$
H^{m}(X \times X, \mathbb{Q}) \cong \bigoplus_{p+q=m} \operatorname{Hom}\left(H^{2 n-p}(X, \mathbb{Q}), H^{q}(X, \mathbb{Q})\right)
$$

On the other hand, we have the following lemma (cf. [32, I, 11.3.3]):

Lemma 2.2. - Let $m=p+q$ be even. A cohomology class

$$
\alpha \in \operatorname{Hom}\left(H^{2 n-p}(X, \mathbb{Q}), H^{q}(X, \mathbb{Q})\right) \subset H^{m}(X \times X, \mathbb{Q})
$$

is a Hodge class on $X \times X$ if and only if it is a morphism of Hodge structures.

There are two kinds of particularly interesting Hodge classes on $X \times X$ obtained from Lemma 2.2.

a) Let $m=2 n$ and consider for each $0 \leq q \leq 2 n$ the element $\operatorname{Id}_{H^{q}(X, \mathbb{Q})}$ which provides by (2) and Lemma 2.2 a Hodge class $\delta_{q}$ of degree $2 n$ on $X$. This class is called the $q$-th Künneth component of the diagonal of $X$. The first standard conjecture (or Künneth's conjecture, cf. [13]) is the following:

Conjecture 2.3. - The classes $\delta_{i}$ are algebraic, that is, are classes of algebraic cycles on $X \times X$ with rational coefficients.

$4^{\text {e }}$ SÉRIE - TOME $46-2013$ - No 3 
b) Let $L$ be an ample line bundle on $X$, and $l:=c_{1}(L) \in H^{2}(X, \mathbb{Q})$. For any integer $k \leq n$, the hard Lefschetz theorem [32, I, 6.2.3] says that the cup-product map

$$
l^{n-k} \cup: H^{k}(X, \mathbb{Q}) \rightarrow H^{2 n-k}(X, \mathbb{Q})
$$

is an isomorphism. This is clearly an isomorphism of Hodge structures. Its inverse

$$
\left(l^{n-k} \cup\right)^{-1}: H^{2 n-k}(X, \mathbb{Q}) \rightarrow H^{k}(X, \mathbb{Q})
$$

is also an isomorphism of Hodge structures, which by (2) and Lemma 2.2 provides a Hodge class $\lambda_{n-k}$ of degree $2 k$ on $X \times X$. The second standard conjecture we will consider (this is one form of Lefschetz' conjecture, cf. [13]) is the following:

Conjecture 2.4. - The classes $\lambda_{i}$ are algebraic, that is, are classes of algebraic cycles on $X \times X$ with rational coefficients.

REMARK 2.5. - One could also ask whether there is a codimension $n$ algebraic cycle $Z$ on $X \times X$ with rational coefficients such that the induced morphism $[Z]_{*}: H^{2 n-k}(X, \mathbb{Q}) \rightarrow$ $H^{k}(X, \mathbb{Q})$ is equal to $\lambda_{k}$. However, if this weaker version is true for any $k$, the Künneth decomposition is algebraic and then by taking the Künneth component of bidegree $(k, k)$ of $Z$, we get an affirmative answer to Conjecture 2.4.

Let us show the following

Proposition 2.6. - The Lefschetz conjecture for all smooth projective varieties $X$ is equivalent to the conjunction of the Künneth Conjecture 2.3 and of Conjecture 1.6 for all smooth projective varieties $X$.

Proof. - Let us assume that the Künneth conjecture holds for $X$ and Conjecture 1.6 holds for any pair $Y \subset X^{\prime}$. Let $i<n$. Consider the Künneth component $\delta_{2 n-i}$ of $\Delta_{X}$, so $\delta_{2 n-i} \in H^{i}(X, \mathbb{Q}) \otimes H^{2 n-i}(X, \mathbb{Q})$ is the class of an algebraic cycle $Z$ on $X \times X$. Let $Y_{i} \stackrel{j_{i}}{\longrightarrow} X$ be a smooth complete intersection of $n-i$ ample hypersurfaces in $X$. Then the Lefschetz theorem on hyperplane sections (cf. [32, II, 1.2.2]) says that

$$
j_{i *}: H^{i}\left(Y_{i}, \mathbb{Q}\right) \rightarrow H^{2 n-i}(X, \mathbb{Q})
$$

is surjective. It follows that the class of the cycle $Z$ vanishes on $X \times\left(X \backslash Y_{i}\right)$. By Conjecture 1.6, there is a $n$-cycle $Z^{\prime}$ supported on $X \times Y_{i}$ such that the class $(i d, j)_{*}\left[Z^{\prime}\right]$ is equal to $[Z]$. Consider the morphism of Hodge structures induced by $\left[Z^{\prime}\right]$ :

$$
\left[Z^{\prime}\right]_{*}: H^{2 n-i}(X, \mathbb{Q}) \rightarrow H^{i}\left(Y_{i}, \mathbb{Q}\right) .
$$

Composing with the morphism $j_{i *}: H^{i}\left(Y_{i}, \mathbb{Q}\right) \rightarrow H^{2 n-i}(X, \mathbb{Q})$, we get $j_{i *} \circ\left[Z^{\prime}\right]_{*}=$ $\operatorname{Id}_{H^{2 n-i}(X, \mathbb{Q})}$. It follows that $\left[Z^{\prime}\right]_{*}$ is injective, and that its transpose $\left[Z^{\prime}\right]^{*}: H^{i}\left(Y_{i}, \mathbb{Q}\right) \rightarrow$ $H^{i}(X, \mathbb{Q})$ is surjective. We now apply [4, Proposition 8] and induction on dimension to conclude that Lefschetz' conjecture holds for $X$.

Conversely, assume Lefschetz' conjecture holds for any smooth projective variety. It obviously implies the Künneth conjecture. It is a well-known fact (cf. [13], [34, Theorem 4]) that the Lefschetz standard conjecture for a smooth complex projective variety $M$ implies that numerical and homological equivalences coincide for cycles on $M$. Let us show, following a suggestion of the referee simplifying our original proof, that the conclusion of Conjecture 1.6 
for $X, Y, Z$ satisfying the given assumptions is already implied by the fact that homological and numerical equivalences coincide for cycles on $X$ and a desingularization $\widetilde{Y}$ of $Y$. Set $n=\operatorname{dim} X, k=\operatorname{codim} Z, l=\operatorname{codim} Y$. Let $\tilde{j}: \widetilde{Y} \rightarrow X$ be a desingularization of $Y$. It is known (cf. [34, Proposition 3]) that there exists a class $\beta \in H^{2 k-2 l}(\widetilde{Y}, \mathbb{Q})$ such that $\tilde{j}_{*} \beta=[Z]$. This class $\beta$ gives by intersection a linear form $\eta$ on the space $H^{2 n-2 k}(\widetilde{Y}, \mathbb{Q})_{\text {alg. }}$. If homological and numerical equivalences coincide for cycles on $\widetilde{Y}$, there is a codimension $k-l$ cycle $Z^{\prime}$ with $\mathbb{Q}$-coefficients on $Y$, such that $\eta$ is given by intersecting with the class of $Z^{\prime}$. The class $j_{*}\left[Z^{\prime}\right] \in H^{2 k}(X, \mathbb{Q})$ has the property that for any codimension $n-k$ cycle class $\gamma$ on $X$,

$$
<j_{*}\left[Z^{\prime}\right], \gamma>_{X}=<\left[Z^{\prime}\right], j^{*} \gamma>_{\widetilde{Y}}=<\beta, j^{*} \gamma>_{\widetilde{Y}}=<j_{*} \beta, \gamma>_{X}=<[Z], \gamma>_{X} .
$$

Thus, if homological and numerical equivalences coincide for cycles on $X$, we conclude that $[Z]=j_{*}\left[Z^{\prime}\right]$.

The main use we will make of Conjecture 1.6 is the following strengthening of the generalized Hodge conjecture. Let $X$ be a smooth complex projective variety of dimension $n$, and let $L$ be a sub-Hodge structure of $H^{n}(X, \mathbb{Q})_{\text {prim }}$, where the subscript "prim" stands for "primitive with respect to a given polarization on $X$ ". We know then by the second HodgeRiemann bilinear relations [32, I, 6.3.2] that the intersection form $<$, $>$ restricted to $L$ is nondegenerate. Let $\pi_{L}: H^{n}(X, \mathbb{Q}) \rightarrow L$ be the orthogonal projector on $L$. We assume that $\pi_{L}$ is algebraic, that is, there is a $n$-cycle $\Delta_{L} \subset X \times X$, such that

$$
\begin{aligned}
& {\left[\Delta_{L}\right]_{*}=\pi_{L}: H^{n}(X, \mathbb{Q}) \rightarrow L \subset H^{n}(X, \mathbb{Q}),} \\
& {\left[\Delta_{L}\right]_{*}=0: H^{i}(X, \mathbb{Q}) \rightarrow H^{i}(X, \mathbb{Q}), i \neq n .}
\end{aligned}
$$

Lemma 2.7. - Assume that there exists a closed algebraic subset $Y \subset X$ such that $L$ vanishes in $H^{n}(X \backslash Y, \mathbb{Q})$. Then if Conjecture 1.6 holds, there is a cycle $Z_{L}^{\prime} \subset Y \times Y$ with $\mathbb{Q}$-coefficients such that

$$
\left[Z_{L}^{\prime}\right]=\left[\Delta_{L}\right] \text { in } H^{2 n}(X \times X, \mathbb{Q}) .
$$

Proof. - Indeed, because $\pi_{L}$ is the orthogonal projector on $L$, the class $\left[Z_{L}\right]$ belongs to $L \otimes L \subset H^{2 n}(X \times X, \mathbb{Q})$. As $L$ vanishes in $H^{n}(X \backslash Y, \mathbb{Q})$, the class $\left[Z_{L}\right] \in L \otimes L$ vanishes in $H^{2 n}(X \times X \backslash(Y \times Y), \mathbb{Q})$. Conjecture 1.6 then guarantees the existence of a cycle $Z_{L}^{\prime} \subset Y \times Y$ such that $\left[Z_{L}^{\prime}\right]=\left[\Delta_{L}\right]$ in $H^{2 n}(X \times X, \mathbb{Q})$.

\section{Proof of Theorem 1.7}

\subsection{Generalities on varieties with "trivial" Chow groups}

We will say that a (non necessarily projective) smooth variety satisfies property $\mathscr{P}$ (or has trivial Chow groups) if the cycle map

$$
\mathrm{cl}: C H^{i}(X)_{\mathbb{Q}} \rightarrow H^{2 i}(X, \mathbb{Q})
$$

is injective for all $i$. We will say that $X$ satisfies property $\mathscr{P}_{k}$ if the cycle class map

$$
\mathrm{cl}: C H^{i}(X)_{\mathbb{Q}} \rightarrow H^{2 i}(X, \mathbb{Q})
$$

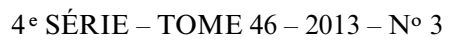


is injective for all $i \leq k$. When $X$ is projective, it is known (cf. [14], [16]) that if $X$ has trivial Chow groups, the cycle class map

$$
\mathrm{cl}: C H^{k}(X)_{\mathbb{Q}} \rightarrow H^{2 k}(X, \mathbb{Q})
$$

is an isomorphism for any $k$ and that $H^{2 k+1}(X, \mathbb{Q})=0$ for all $k$. We have the following lemma:

Lemma 3.1. - Assume Conjecture 1.6. Let $X$ be a smooth algebraic variety satisfying property $\mathscr{P}$. Then any Zariski open set $U \subset X$ satisfies property $\mathscr{P}$.

Proof. - Write $U=X \backslash Y$. Let $Z$ be a codimension $k$ cycle on $U$ with vanishing cohomology class. Then $Z$ is the restriction to $U$ of a cycle $\bar{Z}$ on $X$, which has the property that

$$
[\bar{Z}]_{\mid U}=0
$$

Conjecture 1.6 says that there is a cycle $Z^{\prime}$ supported on $Y$ such that $[\bar{Z}]=\left[Z^{\prime}\right]$ in $H^{2 k}(X, \mathbb{Q})$. The cycle $\bar{Z}-Z^{\prime}$ is thus cohomologous to 0 on $X$. As $X$ satisfies property $\mathscr{P}, \bar{Z}-Z^{\prime}$ is rationally equivalent to 0 on $X$ modulo torsion, and so is its restriction to $U$, which is equal to $Z$.

Lemma 3.2. - Let $X$ be a smooth complex variety satisfying property $\mathscr{P}_{k}$. Then any projective bundle $p: \mathbb{P}(E) \rightarrow X$, where $E$ is a locally free sheaf on $X$, satisfies property $\mathscr{P}_{k}$.

Proof. - Indeed, let $h=c_{1}\left(\Theta_{\mathbb{P}(E)}(1)\right) \in C H^{1}(\mathbb{P}(E))$ and let $[h] \in H^{2}(\mathbb{P}(E), \mathbb{Q})$ be its topological first Chern class. The canonical decompositions ([32, I,7.3.3], [32, II,9.3.2]

$$
\begin{aligned}
C H^{*}(\mathbb{P}(E))_{\mathbb{Q}} & =\oplus_{0 \leq i \leq r-1} h^{i} p^{*} C H^{*-i}(X, \mathbb{Q}), \\
H^{*}(\mathbb{P}(E))_{\mathbb{Q}} & =\oplus_{0 \leq i \leq r-1}[h]^{i} \cup p^{*} H^{*-2 i}(X, \mathbb{Q}),
\end{aligned}
$$

are compatible with the cycle map cl :CH* $(X) \rightarrow H^{2 *}(X, \mathbb{Q})$. Thus if cl is injective on cycles of codimension $\leq k$ on $X$, it is also injective on cycles of codimension $\leq k$ on $\mathbb{P}(E)$.

We prove similarly

Lemma 3.3. - Let $X$ be a smooth complex algebraic variety satisfying property $\mathscr{P}_{k}$ and let $Y \subset X$ be a subvariety satisfying property $\mathscr{D}_{k-1}$. Then the blow-up $\widetilde{X}_{Y} \rightarrow X$ of $X$ along $Y$ satisfies property $\mathscr{P}_{k}$.

Let us conclude with two more properties:

Lemma 3.4. - Let $\phi: X \rightarrow X^{\prime}$ be a projective surjective morphism, where $X$ and $X^{\prime}$ are smooth complex algebraic varieties. If $X$ satisfies property $\mathscr{P}$, so does $X^{\prime}$.

Proof. - Indeed, let $h \in C H^{1}(X)$ be the first Chern class of a relatively ample line bundle. Let $r=\operatorname{dim} X-\operatorname{dim} X^{\prime}$, and let $d$ be defined by $\phi_{*} h^{r}=d X^{\prime} \in C H^{0}\left(X^{\prime}\right)$. Then we have the projection formula:

$$
\phi_{*}\left(h^{r} \cdot \phi^{*} \alpha\right)=d \alpha, \forall \alpha \in C H^{*}\left(X^{\prime}\right)_{\mathbb{Q}}
$$

If $\alpha \in C H^{*}\left(X^{\prime}\right)_{\mathbb{Q}}$ satisfies $\operatorname{cl}(\alpha)=0$ then $\phi^{*}(\operatorname{cl}(\alpha))=\operatorname{cl}\left(\phi^{*} \alpha\right)=0$ in $H^{2 *}(X, \mathbb{Q})$. Thus $\phi^{*} \alpha=0$ in $C H^{*}(X)_{\mathbb{Q}}$ and $\alpha=0$ in $C H\left(X^{\prime}\right)_{\mathbb{Q}}$ by (3). 
Proposition 3.5. - Let $X$ be a smooth projective variety satisfying property $\mathscr{D}$. Then $X \times X$ satisfies property $\mathscr{P}$.

Proof. - This uses the fact (proved e.g. in [22]) that a variety satisfying property $\mathscr{P}$ has a complete decomposition of the diagonal as a combination of products of algebraic cycles (cf. also [32, II,10.3.1]):

$$
\Delta_{X}=\sum_{i, j} n_{i j} Z_{i} \times Z_{j} \text { in } C H^{n}(X \times X)_{\mathbb{Q}}
$$

where $n_{i j} \in \mathbb{Q}$, and $\operatorname{dim} Z_{i}+\operatorname{dim} Z_{j}=n=\operatorname{dim} X$. It follows that the variety $Z:=X \times X$ also admits such decomposition, since $\Delta_{Z}=p_{13}^{*} \Delta_{X} \cdot p_{24}^{*} \Delta_{X}$ in $C H^{2 n}(Z \times Z)$, where $p_{i j}$ is the projection of $Z \times Z=X^{4}$ to the product $X \times X$ of the $i$-th and $j$-th summand.

But this in turn implies that $C H^{*}(Z)_{\mathbb{Q}} \cong H^{2 *}(Z, \mathbb{Q})$. Indeed, write

$$
\Delta_{Z}=\sum_{i, j} m_{i j} W_{i} \times W_{j} \text { in } C H^{2 n}(Z \times Z) .
$$

Then any cycle $\gamma \in C H(Z)_{\mathbb{Q}}$ satisfies

$$
\gamma=\Delta_{Z *} \gamma=\sum_{i, j} m_{i j} \operatorname{deg}\left(\gamma \cdot W_{i}\right) W_{j} \text { in } C H(Z)_{\mathbb{Q}} .
$$

It immediately follows that if $\gamma$ is homologous to 0 , it vanishes in $C H(Z)_{\mathbb{Q}}$.

\subsection{Proof of Theorem 1.7}

We will start with a few preparatory lemmas. Consider a smooth projective variety $X$ of dimension $n$ satisfying property $\mathscr{P}$. Let $\mathscr{L}_{1}, \ldots, \mathscr{L}_{r}$ be very ample line bundles on $X$. Let $j: X_{b} \hookrightarrow X$ be a general complete intersection of members of $\left|\mathscr{L}_{i}\right|$. Then $X_{b}$ is smooth of dimension $n-r$, and we have an orthogonal decomposition

$$
H^{n-r}\left(X_{b}, \mathbb{Q}\right)=H^{n-r}\left(X_{b}, \mathbb{Q}\right)_{\operatorname{van}} \oplus H^{n-r}(X, \mathbb{Q})_{\mid X_{b}},
$$

where the vanishing cohomology $L_{b}:=H^{n-r}\left(X_{b}, \mathbb{Q}\right)_{\text {van }}$ is defined as

$$
\operatorname{Ker}\left(j_{*}: H^{n-r}\left(X_{b}, \mathbb{Q}\right) \rightarrow H^{n+r}(X, \mathbb{Q})\right) .
$$

Note that $H^{n-r}\left(X_{b}, \mathbb{Q}\right)_{\text {van }}$ is contained in $H^{n-r}\left(X_{b}, \mathbb{Q}\right)_{\text {prim }}$, where "prim" means primitive for the polarization $c_{1}\left(\mathscr{L}_{1}\right)_{\mid X_{b}}$, and thus, by the second Hodge-Riemann bilinear relations, the intersection form $<,>$ on $H^{n-1}\left(X_{b}, \mathbb{Q}\right)$ remains nondegenerate after restriction to $L_{b}$.

Next the Lefschetz theorem on hyperplane sections says that the restriction maps $H^{i}(X, \mathbb{Q}) \rightarrow H^{i}\left(X_{b}, \mathbb{Q}\right)$ are isomorphisms for $i<n-r$. Furthermore, the hard Lefschetz theorem applied to $X_{b}$ says that denoting by $h$ the restriction to $X_{b}$ of the first Chern class of an ample line bundle $H$ on $X$, the cup-product map

$$
h^{k} \cup: H^{n-r-k}\left(X_{b}, \mathbb{Q}\right) \rightarrow H^{n-r+k}\left(X_{b}, \mathbb{Q}\right)
$$

is surjective for any $k \geq 0$. It follows that the restriction maps $H^{i}(X, \mathbb{Q}) \rightarrow H^{i}\left(X_{b}, \mathbb{Q}\right)$ are also surjective for $i>n-r$.

All the above statements remain valid if instead of a complete intersection of very ample hypersurfaces we consider the zero set $X_{b}$ of a section of an ample vector bundle $E$. For example, the Lefschetz theorem on hyperplane sections has to be replaced by Sommese's theorem [27] which applies to sections of ample vector bundles. As for the statement that 
the intersection form $<,>$ on $H^{n-r}\left(X_{b}, \mathbb{Q}\right)$ remains nondegenerate after restriction to $L_{b}=H^{n-r}\left(X_{b}, \mathbb{Q}\right)_{\text {van }}$, it is a consequence of the following construction of Terasoma (cf. [28]). The section $\sigma_{b}$ defining $X_{b}$ defines as well an hypersurface $Y_{b} \subset \mathbb{P}(E)$, which is a member of the very ample linear system $\left|\Theta_{\mathbb{P}(E)}(1)\right|$. There is a correspondence between $X_{b}$ and $Y_{b}$ which comes from the fact that $Y_{b}$ contains the projective bundle $Y_{b}^{\prime}:=\mathbb{P}\left(E_{\mid X_{b}}\right)$. Let $j_{Y_{b}^{\prime}}: Y_{b}^{\prime} \rightarrow Y_{b}$ be the inclusion, and $\pi_{Y_{b}^{\prime}}: Y_{b}^{\prime} \rightarrow X_{b}$ be the structural map. Then we get a map

$$
j_{Y_{b}^{\prime} *} \circ \pi_{Y_{b}^{\prime}}: H^{n-r}\left(X_{b}, \mathbb{Q}\right) \rightarrow H^{n+r-2}\left(Y_{b}, \mathbb{Q}\right)
$$

which sends $H^{n-r}\left(X_{b}, \mathbb{Q}\right)_{\text {van }}$ in $H^{n+r-2}\left(Y_{b}, \mathbb{Q}\right)_{\text {van }}$ and preserves the cup-product up to sign. The nondegeneracy of the cup-product restricted to $H^{n-r}\left(X_{b}, \mathbb{Q}\right)_{\text {van }}$ then follows from the fact that the cup-product polarizes the Hodge structure on $H^{n+r-2}\left(Y_{b}, \mathbb{Q}\right)_{\text {van }}$.

As $X$ has trivial Chow groups, we know by [16] that $H^{*}(X, \mathbb{Q})$ is generated by classes of algebraic cycles and so is the restriction $H^{*}(X, \mathbb{Q})_{\mid X_{b}}$, that is the orthogonal complement of $L_{b}$ in $H^{*}\left(X_{b}, \mathbb{Q}\right)$. This implies the following:

\section{Lemma 3.6. - The orthogonal projector $\pi_{L_{b}}$ on $L_{b}$ is algebraic.}

Proof. - In fact, having chosen an ample line bundle $H$ on $X$, we can construct a canonical algebraic cycle $\Delta_{b \text {,van }}$ with $\mathbb{Q}$-coefficients on $X_{b} \times X_{b}$, whose class $\left[\Delta_{b \text {,van }}\right]$ is equal to $\pi_{L_{b}}$. For this, we choose a basis of $\oplus_{i \leq n-r} H^{2 i}(X, \mathbb{Q})$. As we know that $X$ has trivial Chow groups, this basis consists of classes $\left[z_{i, j}\right]$ of algebraic cycles $z_{i, j}$ on $X$, with codim $z_{i, j}=i \leq n-r$. With the same notation $h:=c_{1}\left(H_{\mid X_{b}}\right)$, we find that the classes $h^{n-r-2 i} \cup\left[z_{i, j}\right]_{\mid X_{b}}$, together with the classes $\left[z_{i, j}\right]_{\mid X_{b}}$, form a basis of $H^{*}(X, \mathbb{Q})_{\mid X_{b}}$. These classes are the restrictions to $X_{b}$ of the canonically defined algebraic cycles $H^{n-r-2 i} \cdot z_{i, j}$ of $X$.

The intersection form on $H^{*}\left(X_{b}, \mathbb{Q}\right)$ is nondegenerate when restricted to $H^{*}(X, \mathbb{Q})_{\mid X_{b}}$, and $L_{b}$ is the orthogonal complement of $H^{*}(X, \mathbb{Q})_{\mid X_{b}}$ with respect to the intersection pairing on $H^{*}\left(X_{b}, \mathbb{Q}\right)$. We thus have the equality of orthogonal projectors:

$$
\pi_{L_{b}}+\pi_{H^{*}(X, \mathbb{Q})_{\mid X_{b}}}=\operatorname{Id}_{H^{*}\left(X_{b}, \mathbb{Q}\right)} .
$$

But it is clear that the orthogonal projector $\pi_{H^{*}(X, \mathbb{Q})_{\mid X_{b}}}$ is given by the class of a canonical algebraic cycle on $X_{b} \times X_{b}$ (it suffices to construct from the previous basis an orthogonal basis $\left(e_{i}\right)$ of $H^{*}(X, \mathbb{Q})_{\mid X_{b}}$ for the intersection form on $H^{*}\left(X_{b}, \mathbb{Q}\right)$. This allows to write $\pi_{H^{*}(X, \mathbb{Q})_{\mid X_{b}}}$ as the element $\sum_{i} \lambda_{i} e_{i} \otimes e_{i}$ of $H^{*}(X, \mathbb{Q})_{\mid X_{b}} \otimes H^{*}(X, \mathbb{Q})_{\mid X_{b}} \subset \operatorname{End} H^{*}\left(X_{b}, \mathbb{Q}\right)$ and one concludes using the fact that the $e_{i}$ are by construction classes of canonically defined algebraic cycles $)$. As $\operatorname{Id}_{H^{*}\left(X_{b}, \mathbb{Q}\right)}$ corresponds to the class of the diagonal of $X_{b}$, the proof is finished.

We now assume that there is a closed algebraic subset $Y_{b} \subset X_{b}$ of codimension $c$ such that $L_{b}$ vanishes on $X_{b} \backslash Y_{b}$. Then, under Conjecture 1.6, Lemma 2.7 tells that there is an algebraic cycle $Z_{b}$ supported on $Y_{b} \times Y_{b}$ such that $\left[Z_{b}\right]=\left[\Delta_{b, \text { van }}\right]$.

The key point now is the following Proposition 3.7. In the following, we will put everything in family. Let $\pi: \chi \rightarrow B$ be a smooth projective morphism and let $(\pi, \pi): \chi \times_{B} \chi \rightarrow B$ be the fibered self-product of $\chi$ over $B$. Let $Z \subset \chi \times_{B} \chi$ be a codimension $k$ algebraic cycle. We denote the fibres $\chi_{b}:=\pi^{-1}(b), Z_{b}:=Z_{\mid \chi_{b} \times \chi_{b}}$. 
Proposition 3.7. - Assume that for a very general point $b \in B$, there exist a closed algebraic subset $Y_{b} \subset \chi_{b} \times \chi_{b}$ of codimension $c$, and an algebraic cycle $Z_{b}^{\prime} \subset Y_{b} \times Y_{b}$ with $\mathbb{Q}$-coefficients, such that

$$
\left[Z_{b}^{\prime}\right]=\left[Z_{b}\right] \text { in } H^{2 k}\left(\chi_{b} \times \chi_{b}, \mathbb{Q}\right) .
$$

Then there exist a closed algebraic subset $Y \subset \chi$ of codimension $c$, and a codimension $k$ algebraic cycle $Z^{\prime}$ with $\mathbb{Q}$-coefficients on $\chi \times_{B} \chi$, which is supported on $\mathcal{Y} \times_{B} Y$ and such that for any $b \in B$,

$$
\left[Z_{b}^{\prime}\right]=\left[Z_{b}\right] \text { in } H^{2 k}\left(\chi_{b} \times \chi_{b}, \mathbb{Q}\right) .
$$

REMARK 3.8. - This proposition is a crucial observation in the present paper. As we will see later, the key point is the fact that we do not need to make any base change for this specific problem, hence we will be able to use the fact that the total spaces $\chi$ and $\chi \times_{B} \chi$ are rather simple. The idea of spreading-out cycles has become very important in the theory of algebraic cycles since Nori's paper [19], (cf. [9], [25]). For most problems however, we usually need to work over a generically finite extension of the base, due to the fact that cycles existing at the general point will exist on the total space of the family only after a base change.

Proof of Proposition 3.7. - There are countably many algebraic varieties $\phi_{i}: M_{i} \rightarrow B$ parameterizing data $\left(b, Y_{b}, Z_{b}^{\prime}\right)$ as above, and we can assume that each $M_{i}$ parameterizes universal objects

$$
y_{i} \rightarrow M_{i}, y_{i} \subset x_{M_{i}}, z_{i}^{\prime} \subset y_{i} \times_{M_{i}} y_{i},
$$

satisfying the property that for $m \in M_{i}$, with $\operatorname{pr}_{1}(m)=b \in B$,

$$
\left[\mathcal{Z}_{i, b}^{\prime}\right]=\left[\mathcal{Z}_{i, b}\right] \text { in } H^{2 k}\left(\chi_{b} \times \chi_{b}, \mathbb{Q}\right) .
$$

By assumption, $B$ is the union of the images of the morphisms $\phi_{i}: M_{i} \rightarrow B$. By a Baire category argument, we conclude that one of the morphisms $\phi_{i}$ is dominating. Taking a subvariety of $M_{i}$ if necessary, we may assume that $\phi_{i}: M_{i} \rightarrow B$ is generically finite. We may also assume that it is proper and carries the families $y_{i} \rightarrow M_{i}, y_{i} \subset \chi_{M_{i}}, Z_{i}^{\prime} \subset \mathcal{Y}_{i} \times M_{i} y_{i}$. Denote by $r_{i}: \chi_{M_{i}} \rightarrow \chi$ the proper generically finite morphism induced by $\phi_{i}$. Let

$$
y:=r_{i}\left(y_{i}\right) \subset \chi \text {. }
$$

Note that because $r_{i}$ is generically finite, $\operatorname{codim} y \leq c$. Let $r_{i}^{\prime}: \mathcal{Y}_{i} \rightarrow \mathcal{Y}$ be the restriction of $r_{i}$ to $Y_{i}$. and let $Z^{\prime}:=\left(r_{i}^{\prime}, r_{i}^{\prime}\right)_{*}\left(Z_{i}^{\prime}\right)$, which is a codimension $k$ cycle in $\chi \times_{B} \chi$ supported in

$$
\left(r_{i}^{\prime}, r_{i}^{\prime}\right)\left(\mathcal{Y}_{i} \times_{M_{i}} \mathscr{Y}_{i}\right) \subset \mathcal{Y} \times_{B} Y .
$$

It is obvious that for any $b \in B,\left[\mathcal{Z}_{b}^{\prime}\right]=N\left[\mathcal{Z}_{b}\right]$ in $H^{2 k}\left(\chi_{b} \times \chi_{b}, \mathbb{Q}\right)$, where $N$ is the degree of $r_{i}$.

In the application, $\chi$ and $B$ will be as in the following :

Notation 3.9. - Let $X$ be a smooth projective variety with trivial Chow groups and $E$ be a very ample vector bundle on $X$ of rank $r$. Let $\mathbb{P}:=\mathbb{P}\left(H^{0}(X, E)\right)$. Let $B \subset \mathbb{P}$ be the open set of sections whose zero set is smooth of codimension $r$, and let

$$
\chi \subset B \times X, \pi: \chi \rightarrow B,
$$

be the universal family. We will denote $X_{b} \subset \chi$ the fibre $\pi^{-1}(b)$ for $b \in B$.

4 e SÉRIE - TOME $46-2013$ - No 3 
We apply the previous proposition to $Z=\mathscr{D}_{\text {van }}$, the corrected relative diagonal with fibre over $b \in B$ the $\Delta_{b \text {,van }}$ introduced in Lemma 3.6. (Note that $\mathscr{D}_{\text {van }}$ is not in fact canonically determined by its restrictions to the fibers $X_{b} \times X_{b}$, as it may be modified by adding cycles which are restrictions to $\chi \times_{B} \chi$ of cycles in $C H^{>0}(B) \otimes C H(X \times X) \subset C H(B \times X \times X)$.)

We then get the following :

Lemma 3.10. - Assume that for a general point $b \in B$, there is a codimension $c$ closed algebraic subset $Y_{b} \subset X_{b}$ such that $H^{n-r}\left(X_{b}, \mathbb{Q}\right)_{\text {van }}$ vanishes on $X_{b} \backslash Y_{b}$. If furthermore Conjecture 1.6 holds, there exist a closed algebraic subset $Y \subset \chi$ of codimension $c$, and a codimension $n-r$ algebraic cycle $Z^{\prime}$ on $\chi \times_{B} \chi$ with $\mathbb{Q}$-coefficients, which is supported on $Y \times_{B} y$ and such that for any $b \in B$,

$$
\left[Z_{b}^{\prime}\right]=\left[\Delta_{b, \mathrm{van}}\right] \text { in } H^{2 n-2 r}\left(X_{b} \times X_{b}, \mathbb{Q}\right) .
$$

Proof. - This is a direct application of Proposition 3.7, because we know from Lemmas 2.7 and 3.6 that under Conjecture 1.6, the assumption implies that there exists for any $b \in B$ an algebraic cycle $Z_{b}^{\prime} \subset Y_{b} \times Y_{b}$ such that $\left[Z_{b}^{\prime}\right]=\left[\Delta_{b, \text { van }}\right]$ in $H^{2 n-2 r}\left(X_{b} \times X_{b}, \mathbb{Q}\right)$.

We have next the following:

Lemma 3.11. - With notation as in 3.9, let $\alpha \in H^{2 n-2 r}\left(\chi \times_{B} \chi, \mathbb{Q}\right)$ be a cohomology class whose restriction to the fibres $X_{b} \times X_{b}$ is 0 . Then we can write

$$
\alpha=\alpha_{1}+\alpha_{2}
$$

where $\alpha_{1}$ is the restriction to $\chi \times_{B} \chi$ of a class $\beta_{1} \in H^{2 n-2 r}(X \times \chi, \mathbb{Q})$, and $\alpha_{2}$ is the restriction to $\chi \times_{B} \chi$ of a class $\beta_{2} \in H^{2 n-2 r}(\chi \times X, \mathbb{Q})$.

More precisely we can take $\beta_{1} \in \oplus_{i<n-r} H^{i}(X, \mathbb{Q}) \otimes L^{1} H^{2 n-2 r-i}(\chi, \mathbb{Q})$, and $\beta_{2} \in \oplus_{i<n-r} L^{1} H^{2 n-2 r-i}(\chi, \mathbb{Q}) \otimes H^{i}(X, \mathbb{Q})$, where $L$ stands for the Leray filtration on $H^{*}(\chi, \mathbb{Q})$ with respect to the morphism $\pi: \chi \rightarrow B$.

Proof. - Consider the smooth proper morphism

$$
(\pi, \pi): \chi \times_{B} \chi \rightarrow B .
$$

The relative Künneth decomposition gives

$$
R^{k}(\pi, \pi)_{*} \mathbb{Q}=\bigoplus_{i+j=k} H_{\mathbb{Q}}^{i} \otimes H_{\mathbb{Q}}^{j},
$$

where $H_{\mathbb{Q}}^{i}:=R^{i} \pi_{*} \mathbb{Q}$. The Leray spectral sequence of $(\pi, \pi)$, which degenerates at $E_{2}$ (cf. [6]), gives the Leray filtration $L$ on $H^{2 n-2 r}\left(\chi \times_{B} \chi, \mathbb{Q}\right)$ with graded pieces

$$
\operatorname{Gr}_{L}^{l} H^{2 n-2 r}\left(\chi \times_{B} \chi, \mathbb{Q}\right)=H^{l}\left(B, R^{2 n-2 r-l}(\pi, \pi)_{*} \mathbb{Q}\right)=\bigoplus_{i+j=2 n-2 r-l} H^{l}\left(B, H_{\mathbb{Q}}^{i} \otimes H_{\mathbb{Q}}^{j}\right) .
$$

Our assumption on $\alpha$ exactly says that it vanishes in the first quotient $H^{0}\left(B, R^{2 n-2 r}(\pi, \pi)_{*} \mathbb{Q}\right)$, or equivalently, $\alpha \in L^{1} H^{2 n-2 r}\left(\chi \times_{B} \chi, \mathbb{Q}\right)$. Consider now the other graded pieces

$$
H^{l}\left(B, H_{\mathbb{Q}}^{i} \otimes H_{\mathbb{Q}}^{j}\right), l>0, i+j=2 n-2 r-l .
$$

Since $l>0$, and $i+j=2 n-2 r-l$, we have either $i<n-r$ or $j<n-r$. Let us consider the case where $i<n-r$ : then the Lefschetz hyperplane section theorem tells that the sheaf $H_{\mathbb{Q}}^{i}$ is the constant sheaf with stalk $H^{i}(X, \mathbb{Q})$. Thus we find that 
$H^{l}\left(B, H_{\mathbb{Q}}^{i} \otimes H_{\mathbb{Q}}^{j}\right)=H^{i}(X, \mathbb{Q}) \otimes H^{l}\left(B, H_{\mathbb{Q}}^{j}\right)$, which is a Leray graded piece of $H^{i}(X, \mathbb{Q}) \otimes H^{l+j}(\chi)$. Analyzing similarly the case where $j<n-r$, we conclude that the natural map

$$
\begin{gathered}
\bigoplus_{i<n-r} H^{i}(X, \mathbb{Q}) \otimes L^{1} H^{2 n-2 r-i}(\chi, \mathbb{Q}) \oplus \bigoplus_{j<n-r} L^{1} H^{2 n-2 r-j}(\chi, \mathbb{Q}) \otimes H^{j}(X, \mathbb{Q}) \\
\rightarrow L^{1} H^{2 n-2 r}\left(\chi \times_{B} \chi, \mathbb{Q}\right)
\end{gathered}
$$

is surjective. This proves the existence of the classes $\beta_{1}, \beta_{2}$.

In the case where $X$ has trivial Chow groups, we get extra information:

Lemma 3.12. - With the same notations as above, assume that $X$ has trivial Chow groups and that $\alpha$ is the class of an algebraic cycle on $\chi_{\times_{B}} \chi$. Then, up to shrinking $B$ to a dense Zarisk $i$ open set, we can choose the $\beta_{i}$ 's to be the restriction of classes of algebraic cycles on $B \times X \times X$.

Proof. - We first claim that it suffices to show that, up to shrinking $B$ if necessary, we can choose $\beta_{1}$, resp. $\beta_{2}$ to be the class of an algebraic cycle on $X \times \mathcal{X}$, resp on $\chi \times X$. Indeed, $\chi$ is a Zariski open set in $\mathbb{P}^{\prime}$, where $\mathbb{P}^{\prime} \subset \mathbb{P} \times X$ is defined as follows :

$$
\mathbb{P}^{\prime}:=\{(\sigma, x), \sigma(x)=0\} .
$$

Via the second projection, $\mathbb{P}^{\prime}$ is a projective bundle over $X$ with fiber over $x$ the codimension $r$ subspace $\mathbb{P}_{x}^{\prime} \subset \mathbb{P}$ of sections vanishing at $x$, because we assumed $E$ is globally generated.

It follows that $X \times \chi$ is as well a Zariski open set in the corresponding fibration $X \times \mathbb{P}^{\prime} \rightarrow X \times X$ into projective spaces. The restriction map

$$
C H(X \times X \times \mathbb{P}) \rightarrow C H\left(X \times \mathbb{P}^{\prime}\right)
$$

is then surjective, by the computation of the Chow groups of a projective bundle fibration ([32, II,9.3.2]) and thus, composing with the restriction to the Zariski open set $X \times \chi$, we get that the restriction map

$$
C H(X \times X \times \mathbb{P}) \rightarrow C H(X \times \chi)
$$

is also surjective. It follows that the restriction map $C H(X \times X \times B) \rightarrow C H(X \times \chi)$ is also surjective, so that if we can choose $\beta_{1}$ to be algebraic on $X \times \chi$, then it lifts to an algebraic cycle class on $X \times X \times B$. We argue similarly for $\beta_{2}$.

We know that the cohomology of $X$ is generated by classes of algebraic cycles $\left[z_{i, j}\right] \in H^{2 i}(X, \mathbb{Q})$. Let us choose a basis of $H^{*<n-r}(X, \mathbb{Q})$ consisting of cycle classes $\left[z_{i, j}\right], 2 i<n-r$. Then we can choose cycle classes $\left[z_{i, j}\right]^{*} \in H^{2 n-2 r-2 i}(X, \mathbb{Q})$ in such a way that the restricted classes $\left[z_{i, j}\right]_{\mid X_{b}}^{*}$ form the dual basis of $H^{*>n-r}\left(X_{b}, \mathbb{Q}\right)$. For $i=1,2$, denote by $p_{i, X}: \chi \times_{B} \chi \rightarrow X$ the composition of the inclusion $\chi \times_{B} \chi \hookrightarrow B \times X \times X$ and the projection to the $i$-th $X$-summand.

Observe that for every $i$ such that $2 i<n-r$, the cycle classes

$$
\sum_{j} p_{1, X}^{*}\left[z_{i, j}\right] \cup p_{2, X}^{*}\left[z_{i, j}\right]^{*} \in H^{2 n-2 r}\left(\chi \times_{B} \chi, \mathbb{Q}\right)
$$

seen as cohomological relative self-correspondences of $\chi$ over $B$, provide (maybe up to shrinking $B$ ) projectors

$$
\pi_{2 i}: R \pi_{*} \mathbb{Q} \rightarrow R \pi_{*} \mathbb{Q}
$$

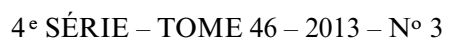


which act as the identity on the cohomology $R^{2 i} \pi_{*} \mathbb{Q}$, for $2 i<n-r$. Similarly the cycle classes

$$
\sum_{j} p_{1, X}^{*}\left[z_{i, j}\right]^{*} \cup p_{2, X}^{*}\left[z_{i, j}\right] \in H^{2 n-2 r}\left(\chi \times_{B} \chi, \mathbb{Q}\right)
$$

give projectors $\pi_{2 n-2 r-2 i}$ of $R \pi_{*} \mathbb{Q}$ acting as the identity on $R^{2 n-2 r-2 i} \pi_{*} \mathbb{Q}$ for $2 i<n-r$. Furthermore, these projectors satisfy the condition that $\pi_{k} \circ \pi_{l}=\pi_{l} \circ \pi_{k}=0$ for $k \neq l$. It follows that denoting

$$
\pi_{n-r}:=\mathrm{Id}-\sum_{2 i<n-r} \pi_{2 i}-\sum_{2 i<n-r} \pi_{2 n-2 r-2 i}
$$

we get a decomposition in the derived category of $B$ :

$$
R \pi_{*} \mathbb{Q} \cong \oplus_{i} R^{i} \pi_{*} \mathbb{Q}[-i],
$$

which in turn induces a similar decomposition by the relative Künneth decomposition :

$$
R(\pi, \pi)_{*} \mathbb{Q} \cong \oplus_{i} R^{i}(\pi, \pi)_{*} \mathbb{Q}[-i]=\oplus_{p+q=i}\left(R^{p} \pi_{*} \mathbb{Q} \otimes R^{q} \pi_{*} \mathbb{Q}\right)[-i] .
$$

Taking cohomology on both sides, we get a decomposition

$$
H^{k}\left(\chi \times_{B} \chi, \mathbb{Q}\right) \cong \oplus_{i+p+q=k} H^{i}\left(B, R^{p} \pi_{*} \mathbb{Q} \otimes R^{q} \pi_{*} \mathbb{Q}\right) .
$$

This is nothing but an explicit form of the Deligne decomposition already mentioned, except that it is clear now that the projector to each summand is induced by an algebraic relative self-correspondence of $\chi \times_{B} \chi$, hence sends a cycle class to a cycle class.

Applying (8) to our class $\alpha$ and recalling that $\alpha$ belongs to $L^{1} H^{*}\left(\chi \times_{B} \chi, \mathbb{Q}\right)$, we get that

$$
\alpha=\sum_{i>0, i+p+q=2 n-2 r} \alpha_{i, p, q},
$$

with $\alpha_{i, p, q} \in H^{i}\left(B, R^{p} \pi_{*} \mathbb{Q} \otimes R^{q} \pi_{*} \mathbb{Q}\right)$ being a cycle class on $\chi \times_{B} \chi$. It now suffices to show that each $\alpha_{i, p, q}$ lifts to a cycle class either on $X \times \mathcal{\chi}$ or on $\chi \times X$.

We have $i+p+q=2 n-2 r$ with $i>0$ so either $p<n-r$ or $q<n-r$. Assume $p<n-r$; then $p$ has to be even, $p=2 m$. The sheaf $R^{2 m} \pi_{*} \mathbb{Q}$ is trivial, with basis given by the pull-back to $\chi$ of the classes $\left[z_{2 m, j}\right]$. We can thus write

$$
\alpha_{i, 2 m, 2 n-2 r-2 m-i}=\sum_{i>0, j} p_{1, X}^{*}\left[z_{2 m, j}\right] \cup p r_{2}^{*} \gamma_{i, 2 m, j},
$$

where $\gamma_{i, 2 m, j} \in H^{i}\left(B, R^{2 n-2 r-2 m-i} \pi_{*} \mathbb{Q}\right)$ is a cohomology class on $\chi$. Here $\pi_{2}^{\prime}: \chi \times_{B} \chi \rightarrow \chi$ is the second projection

The class $\alpha_{i, 2 m, 2 n-2 r-2 m-i}$ being algebraic, so is the class $\pi_{2 *}^{\prime}\left(p_{1, X}^{*}\left[z_{2 m, j}\right]^{*} \cup \alpha_{i, 2 m, 2 n-2 r-2 m-i}\right)$ for any $j$. However, we have the equality

$$
\gamma_{i, 2 m, j}=\pi_{2 *}^{\prime}\left(p_{1, X}^{*}\left[z_{2 m, j}\right]^{*} \cup \alpha_{i, 2 m, 2 n-2 r-2 m-i}\right),
$$

which follows from (10), from the projection formula and from the fact that

$$
\begin{aligned}
& \pi_{2 *}^{\prime}\left(p_{1, X}^{*}\left[z_{2 m, j}\right]^{*} \cup p_{1, X}^{*}\left[z_{2 m, k}\right]\right)=0 \text { in } H^{0}(\chi, \mathbb{Q}) \text { for } j \neq k, \\
& \pi_{2 *}^{\prime}\left(p_{1, X}^{*}\left[z_{2 m, j}\right]^{*} \cup p_{1, X}^{*}\left[z_{2 m, k}\right]\right)=1 \text { in } H^{0}(\chi, \mathbb{Q}) \text { for } j=k .
\end{aligned}
$$

Formula (11) obviously implies that the $\gamma_{i, 2 m, j}$ 's are algebraic, hence that $\alpha_{i, 2 m, 2 n-2 r-2 m-i}$ is algebraic by $(10)$. 
Proof of Theorem 1.7. - We keep notation (3.9) and assume now that the vanishing cohomology $H^{n-r}\left(X_{b}, \mathbb{Q}\right)_{\text {van }}$ is supported on a codimension $c$ closed algebraic subset $Y_{b} \subset X_{b}$ for any $b \in B$. Consider the corrected (or vanishing) diagonal $\mathscr{D}_{\text {van }}$, which is a codimension $n-r$ cycle of $\chi \times_{B} \chi$ with $\mathbb{Q}$-coefficients.

By Lemma 3.10, it follows that there exist a codimension $c$ closed algebraic subset $y \subset \chi$ and a codimension $n-r$ cycle $Z$ on $\chi \times_{B} \chi$ with $\mathbb{Q}$-coefficients, which is supported on $\mathcal{Y} \times{ }_{B} \mathcal{Y}$ and such that

$$
\left[\mathcal{Z}_{b}\right]=\left[\mathscr{D}_{\mathrm{van}, b}\right]=\left[\Delta_{b, \mathrm{van}}\right], \forall b \in B .
$$

Thus the class $[Z]-\left[\Delta_{b, \mathrm{van}}\right] \in H^{2 n-2 r}\left(\chi \times_{B} \chi, \mathbb{Q}\right)$ vanishes on the fibers $X_{b} \times X_{b}$.

Using Lemmas 3.11 and 3.12, we conclude that there is a cycle $\Gamma \in C H^{n-r}(B \times X \times X)_{\mathbb{Q}}$ such that

$$
[\mathcal{Z}]=\left[\mathscr{D}_{\mathrm{van}}\right]+\left[\Gamma_{\mid \chi \times_{B} \chi}\right] \text { in } H^{2 n-2 r}\left(\chi \times_{B} \chi, \mathbb{Q}\right) .
$$

Proposition 3.13. - If X has trivial Chow groups and Conjecture 1.6 holds, the cycle class map

is injective.

$$
C H^{*}\left(\chi \times_{B} \chi\right)_{\mathbb{Q}} \rightarrow H^{2 *}\left(\chi \times_{B} \chi, \mathbb{Q}\right)
$$

Proof. - Consider the blow-up $\overline{X \times X}$ of $X \times X$ along the diagonal. Applying Proposition 3.5 and Lemma 3.3, $\overline{X \times X}$ has trivial Chow groups. A point of $\overline{X \times X}$ parameterizes a couple $(x, y)$ of points of $X$, together with a subscheme $z$ of length 2 of $X$, with associated cycle $x+y$. We thus have the following natural variety

$$
\left.Q=\left\{(\sigma, x, y, z), \sigma \in \mathbb{P}, \sigma_{\mid z}\right)=0 \text { in } H^{0}\left(E_{\mid z}\right)\right\} \subset \mathbb{P} \times \overline{X \times X} .
$$

As $E$ is assumed to be very ample, the map $Q \rightarrow \overline{X \times X}$ is a fibration with fibre over $(x, y, z) \in \overline{X \times X}$ a projective space $\mathbb{P}_{z}$ of codimension $2 r$ in $\mathbb{P}$. By Lemma 3.2, $Q$ also has trivial Chow groups. Let $Q_{0} \subset Q$ be the inverse image of $B$ under the projection $Q \rightarrow \mathbb{P}$. Then $Q_{0}$ is Zariski open in $Q$, so by Lemma 3.1, the cycle class map is also injective on cycles of $Q_{0}$ since we assume Conjecture 1.6. Finally, $Q_{0}$ maps naturally to $\chi \times_{B} \chi$ via the map

$$
\prod_{i} \mathbb{P}_{i} \times \overline{X \times X} \rightarrow \prod_{i} \mathbb{P}_{i} \times X \times X
$$

The morphism $Q_{0} \rightarrow \chi \times_{B} \chi$ being projective and dominant, we conclude by Lemma 3.4 that the cycle class map is injective on cycles of $\chi \times_{B} \chi$.

The proof is then finished as follows. From the equality (12) of cohomology classes, we deduce by Proposition 3.13 the following equality of cycles:

$$
Z=\mathscr{D}_{\text {van }}+\Gamma_{\mid \chi \times_{B}} \chi \text { in } C H^{n-r}\left(\chi \times_{B} \chi\right)_{\mathbb{Q}} .
$$

We now fix $b$ and restrict this equality to $X_{b} \times X_{b}$. Then we find

$$
Z_{b}=\Delta_{b, \text { van }}+\Gamma_{\mid X_{b} \times X_{b}}^{\prime} \text { in } C H^{n-r}\left(X_{b} \times X_{b}\right)_{\mathbb{Q}},
$$

where $\Gamma^{\prime} \in C H(X \times X)_{\mathbb{Q}}$ is the restriction of $\Gamma$ to $b \times X \times X$.

Recalling that $\Delta_{b \text {,van }}=\Delta_{X_{b}}+\Gamma_{\mid X_{b} \times X_{b}}^{\prime \prime}$ for some codimension $n-r$-cycle $\Gamma^{\prime \prime}$ with $\mathbb{Q}$-coefficients on $X \times X$, we conclude that

$$
\Delta_{X_{b}}=Z_{b}+\Gamma_{1 \mid X_{b} \times X_{b}}
$$

4 ${ }^{\text {e }}$ SÉRIE - TOME $46-2013$ - No 3 
where $\Gamma_{1} \in C H^{n-r}(X \times X, \mathbb{Q})$ and the cycle $Z_{b}$ is by construction supported on $\mathcal{Y}_{b} \times \mathcal{Y}_{b}$, with $\mathcal{Y}_{b} \subset X_{b}$ of codimension $\geq c$ for general $b$.

Let $z \in C H_{i}\left(X_{b}\right)_{\mathbb{Q}}$, with $i<c$. Then $\left(Z_{b}\right)_{*} z=0$ since we may find a cycle rationally equivalent to $z$ in $X_{b}$ and disjoint from $Y_{b}$. Applying both sides of (14) to $z$ thus gives :

$$
z=\left(\Gamma_{1 \mid X_{b} \times X_{b}}\right)_{*} z \text { in } C H_{i}\left(X_{b}\right)_{\mathbb{Q}} .
$$

But it is obvious that

$$
\left(\Gamma_{1 \mid X_{b} \times X_{b}}\right)_{*}: C H\left(X_{b}\right)_{\mathbb{Q}} \rightarrow C H\left(X_{b}\right)_{\mathbb{Q}}
$$

factors through $j_{b *}: C H\left(X_{b}\right)_{\mathbb{Q}} \rightarrow C H(X)_{\mathbb{Q}}$. Now, if $z$ is homologous to 0 on $X_{b}, j_{b *}(z)$ is homologous to 0 on $X$, and thus it is rationally equivalent to 0 on $X$ because $X$ has trivial Chow groups. Hence we proved that the cycle map with $\mathbb{Q}$-coefficients is injective on $C H_{i}\left(X_{b}\right)_{\mathbb{Q}}$ for $i<c$, which concludes the proof of the theorem for general $b$. In fact (14) is true for any $b \in B$ by specialization (but a blow-up of $B$ might be necessary in order to exhibit a $Y_{b}$ of codimension $\geq c$ ), and thus the theorem is true as well for any $b \in B$.

\subsection{The surface and threefold cases}

We prove in this section the following theorem (Theorem 1.9 of the introduction) saying that in the surface (resp. threefold) case, the results become unconditional (resp. do not use Conjecture 1.6).

TheOREM 3.14. - Let X be a smooth complex projective variety of dimension $n$ with trivial Chow groups. Let $E$ be a very ample vector bundle of rank $r$ on $X$. Then we have:

1) Case $n-r=2$. If the smooth surfaces $X_{b} \subset X$ obtained as zero loci of sections of $E$ have $h^{2,0}\left(X_{b}\right)=0$, then they satisfy $C H_{0}\left(X_{b}\right)=\mathbb{Z}$.

2) Case $n-r=3$. If the degree 3 cohomology of the threefolds $X_{b} \subset X$ is of geometric coniveau 1 (which is also equivalent to the fact that Abel-Jacobi map $C H^{2}\left(X_{b}\right)_{\mathrm{hom}} \rightarrow J^{3}\left(X_{b}\right)$ is surjective) for very general $X_{b}$, then the Chow group $C H_{0}\left(X_{b}\right)$ is equal to $\mathbb{Z}$.

Proof. - 1) Indeed, the assumption $h^{2,0}\left(X_{b}\right)=0$ implies by the Lefschetz theorem on $(1,1)$-classes that the Hodge structure on $H^{2}\left(X_{b}, \mathbb{Q}\right)$ is generated by divisor classes. So, the generalized Hodge conjecture is true in this case. Furthermore, the case of the Conjecture 1.6 we need for the proof of Theorem 1.7 will be satisfied in this case, because it is satisfied by codimension 2 cycles (cf. Lemma 2.1). Finally, $C H_{0}\left(X_{b}\right)_{\mathbb{Q}}=\mathbb{Q}$ implies $\mathrm{CH}_{0}\left(\mathrm{X}_{b}\right)=\mathbb{Z}$ by Roitman's theorem [24].

2) We have to explain why Conjecture 1.6 will be also satisfied in this case. In fact, we use this conjecture for the proof of Theorem 1.7 in two places. First of all, we need it in the proof of Lemma 2.7, which says that if a certain Hodge structure $L_{b} \subset H^{*}\left(X_{b}, \mathbb{Q}\right)$ is supported on a codimension $c$ closed algebraic subset $Y_{b}$, the corresponding projector has a class which comes from the class of a cycle supported in $Y_{b} \times Y_{b}$. This will be satisfied if $\operatorname{dim} X_{b}=3$, $L_{b} \subset H^{3}\left(X_{b}, \mathbb{Q}\right)$ supported on $Y_{b} \times Y_{b}$ because we know that the degree 6 Hodge class of the projector $\pi_{L}$ is supported on the codimension 2 closed algebraic subset $Y_{b} \times Y_{b}$ (or rather a desingularization of it), so that we can apply Lemma 2.1.

The second place is in the proof of Proposition 3.13 because we use there Lemma 3.1, which is itself heavily based on Conjecture 1.6. However, we can also avoid the use of 
Conjecture 1.6 at this place, for the following reason. In the proof of Proposition 3.13, we arrived at the conclusion that the blow-up of $\chi \times_{B} \chi$ along the diagonal is a Zariski open set of a fibration $Q \rightarrow \overline{X \times X}$ into projective spaces over the blow-up of $X \times X$ along the diagonal. The complementary closed algebraic subset $Q^{\prime} \subset Q$ is the blow-up along the diagonal of the fibered self-product $\chi^{\prime} \times_{B^{\prime}} \chi^{\prime}$, where $B^{\prime} \subset \mathbb{P}\left(H^{0}(X, E)\right)$ is the complement of the Zariski open set $B$, and $\chi^{\prime} \rightarrow B^{\prime}$ is the restriction to $B^{\prime}$ of the universal family over $\mathbb{P}\left(H^{0}(X, E)\right)$.

We have a certain degree 6 cycle class $[Z]$ on $Q$ which vanishes on $Q \backslash Q^{\prime}$, and we need to find a cycle $Z^{\prime}$ supported on $Q^{\prime}$ such that $[Z]=\left[Z^{\prime}\right]$ in $H^{6}(Q, \mathbb{Q})$. As already mentioned (cf. [34, Proposition 3]), this implies that there is a degree 4 Hodge class $\beta$ on a desingularization $\widetilde{Q}^{\prime}$ of $Q^{\prime}$ such that $\tilde{j}_{*} \beta=[Z]$ in $H^{6}(Q, \mathbb{Q})$, where $\tilde{j}: \widetilde{Q}^{\prime} \rightarrow Q$ is the natural morphism. (Here we assume $Q^{\prime} \subset Q$ is of pure codimension 1.) Hence, to conclude, we just need to show that the Hodge conjecture is true for degree 4 Hodge classes on $\widetilde{Q}^{\prime}$. As we do not have a good control of $B^{\prime}$ (because in the proof of Lemma 3.12, we needed to shrink $B$ ), $Q^{\prime}$ may have several irreducible components $Q_{0}^{\prime}, Q_{1}^{\prime}, \ldots, Q_{N}^{\prime}$, where the $Q_{i}^{\prime}, i \geq 1$ are supported over irreducible components $B_{i}^{\prime}$ of $B^{\prime}$ whose generic point parameterizes a smooth subvariety $X_{b} \subset X$ of codimension $r$, while $Q_{0}^{\prime}$ is the irreducible component supported over the locus $B_{0}^{\prime} \subset B$ parameterizing singular $X_{b} \subset X$. In fact, the case of $\widetilde{Q}_{0}^{\prime}$ is not really different from the other ones, because the generic point of $B_{0}^{\prime}$ parameterizes a local complete intersection with one node. In fact, for the desingularization $\widetilde{X}_{b}$ of the generic fiber over $b \in B_{0}^{\prime}$, we still have the property that the degree 3 cohomology $H^{3}\left(\widetilde{X}_{b}, \mathbb{Q}\right)$ is of geometric coniveau $\geq 1$, and that the rest of the cohomology is generated by classes of algebraic cycles. Thus the argument below will apply as well to $Q_{0}^{\prime} \rightarrow B_{0}^{\prime}$. Next what happens over a proper Zariski closed subset $B_{i}^{\prime \prime}$ of $B_{i}^{\prime \prime}$ s needs not to be understood because the classes supported there will come from degree $\leq 2$ on the desingularizations of the corresponding $Q_{i}^{\prime \prime}$, and we can apply the Lefschetz theorem on $(1,1)$-classes. By this argument, we can restrict to the smooth locus of $B_{i}^{\prime}$, and then $Q_{i}^{\prime}$ is also smooth.

We look at the Leray spectral sequence for $\pi_{i}: Q_{i}^{\prime} \rightarrow B_{i}^{\prime}$. This fibration has for fiber the blow-up of $\overline{X_{b} \times X_{b}}$ of $X_{b} \times X_{b}$ over the diagonal. The Leray graded pieces of $H^{4}\left(Q_{i}^{\prime}, \mathbb{Q}\right)$ are

$$
H^{0}\left(B_{i}^{\prime}, R^{4} \pi_{i *} \mathbb{Q}\right), H^{1}\left(B_{i}^{\prime}, R^{3} \pi_{i *} \mathbb{Q}\right), H^{2}\left(B_{i}^{\prime}, R^{2} \pi_{i *} \mathbb{Q}\right), H^{3}\left(B_{i}^{\prime}, R^{1} \pi_{i *} \mathbb{Q}\right), H^{4}\left(B_{i}^{\prime}, R^{0} \pi_{i *} \mathbb{Q}\right) .
$$

The term $H^{3}\left(B_{i}^{\prime}, R^{1} \pi_{i *} \mathbb{Q}\right)$ vanishes since $R^{1} \pi_{i *} \mathbb{Q}=0$ by the Lefschetz theorem on hyperplane sections. The Hodge classes in $H^{2}\left(B_{i}^{\prime}, R^{2} \pi_{i *} \mathbb{Q}\right)$ are products of Hodge classes of degree 2 on $B_{i}^{\prime}$ and Hodge classes of degree 2 on fibers, since $R^{2} \pi_{i *} \mathbb{Q}$ is a constant local system generated by Hodge classes, again by the Lefschetz theorem on hyperplane sections or its Sommese version for vector bundles. Similarly for the term $H^{0}\left(B_{i}^{\prime}, R^{4} \pi_{i *} \mathbb{Q}\right)$.

It remains to consider $H^{1}\left(B_{i}^{\prime}, R^{3} \pi_{i *} \mathbb{Q}\right)$ and $H^{4}\left(B_{i}^{\prime}, R^{0} \pi_{i *} \mathbb{Q}\right)$. The Hodge classes in the first term satisfy the Hodge conjecture because we assumed that the fibers of the restricted families $\chi_{i}^{\prime} \rightarrow B_{i}^{\prime}$ have their degree 3 cohomology of geometric coniveau $\geq 1$. It follows that these classes are supported on some hypersurfaces $Q_{i}^{\prime \prime}$ of $Q_{i}^{\prime}$, hence satisfy the Hodge conjecture by Lefschetz theorem on $(1,1)$-classes applied to a desingularization of $Q_{i}^{\prime \prime}$.

We are left with the last term $H^{4}\left(B_{i}^{\prime}, R^{0} \pi_{i *} \mathbb{Q}\right)$. Obviously we cannot say anything about it, but its image in $H^{6}(Q, \mathbb{Q})$ belongs to the subspace $H^{6}\left(\mathbb{P}\left(H^{0}(X, E)\right), \mathbb{Q}\right) \subset H^{6}(Q, \mathbb{Q})$. We 
are thus reduced to prove that for $W=\mathbb{P}\left(H^{0}(X, E)\right)$, for any codimension 3 cycle $Z$ on $W$, and for any Zariski open set $U=W \backslash \cup_{i} B_{i}^{\prime}$ such that $[Z]_{\mid U}=0$ in $H^{6}(U, \mathbb{Q})$, there is a $\mathbb{Q}$-cycle $Z^{\prime}$ supported on $\cup_{i} B_{i}^{\prime}$ such that $\left[Z^{\prime}\right]=[Z]$ in $H^{6}(W, \mathbb{Q})$, which is quite easy.

\section{Variants and further applications}

\subsection{Complete intersections with group action}

Theorem 1.7 applies to general complete intersections in projective space, the relation (1) giving the Hodge coniveau (hence conjecturally the geometric coniveau $c$ ). There are interesting variants coming from the study of complete intersections $X_{b}$ of $r$ hypersurfaces in projective space $\mathbb{P}^{n}$, or in a product of projective spaces, admitting a finite group action. In most cases, the group $G$ acts on the ambient space, and the ideal of $X_{b}$ is globally invariant under $G$. Assuming $G$ acts on $X_{b}$, let $\chi: G \rightarrow \mathbb{Z} / 2 \mathbb{Z}=\{1,-1\}$ be a character of $G$. Then consider the sub-Hodge structure

$$
L^{\chi}=\left\{\alpha \in H^{n-r}\left(X_{b}, \mathbb{Q}\right)_{\text {prim }}, g^{*} \alpha=\chi(g) \alpha, \forall g \in G\right\} \subset H^{n-r}\left(X_{b}, \mathbb{Q}\right)_{\text {prim }} .
$$

In general, it has a larger coniveau than $X_{b}$. For example if $X_{b}$ is a quintic surface in $\mathbb{P}^{3}$, defined by an invariant polynomial under the linearized group action of $G \cong \mathbb{Z} / 5 \mathbb{Z}$ with generator $g$ on $\mathbb{P}^{3}$ given by

$$
g^{*} X_{i}=\zeta^{i} X_{i}, i=0, \ldots, 3,
$$

where $\zeta$ is a nontrivial 5 -th root of unity, then $H^{2}(S, \mathbb{Q})^{\text {inv }}$ has no $(2,0)$-part, hence is of coniveau 1 , while $H^{2,0}(S) \neq 0$ so the coniveau of $H^{2}(S, \mathbb{Q})_{\text {prim }}$ is 0 . The quotient surface $S / G$ is a quintic Godeaux surface (cf. [29]).

Note that the Hodge structure $L^{\chi}$ corresponds to the projector $\frac{1}{|G|} \sum_{g \in G} \chi(g) g^{*}$ acting on $L$, and it is given by the action of the $n-r$-cycle

$$
\Gamma_{\chi}:=\sum_{g \in G} \chi(g) \Delta_{b, \operatorname{van}, g},
$$

where $\Delta_{b, \text { van }, g}=(\operatorname{Id}, g)_{*}\left(\Delta_{b, \text { van }}\right) \in C H_{n-r}\left(X_{b} \times X_{b}\right)_{\mathbb{Q}}$. The generalized Bloch Conjecture 1.3 (extended to motives) predicts the following :

Conjecture 4.1. - Assume $L^{\chi}$ has coniveau $\geq c$. Then the cycle class map is injective on $C H_{i}\left(X_{b}\right)_{\mathbb{Q}}^{\chi}$ for $i<c$.

If $\chi$ is the trivial character, this conjecture is essentially equivalent to the previous one by considering $X / G$ or a desingularization of it. Even in this case, one needs to make assumptions on the linearized group action in order to apply the same strategy as in the proof of Theorem 1.7. The case of non trivial character cannot be reduced to the previous case.

In order to apply a strategy similar to the one applied for the proof of Theorem 1.7, we need some assumptions. Indeed, if the group $G$ is too big, like the automorphisms group of the Fermat hypersurface, there are too few invariant complete intersections to play on the geometry of the universal family $\chi \rightarrow B$ of $G$-invariant complete intersections.

In any case, what we get mimicking the proof of Theorem 1.7 is the following: $X$ is as before a smooth projective variety of dimension $n$ satisfying property $\mathscr{P}$ and $G$ is a finite group acting on $X$. We study complete intersections $X_{b} \subset X$ of $r G$-invariant ample 
hypersurfaces $X_{i} \in\left|L_{i}\right|^{G}$ : Let $G$ act via the character $\chi_{i}$ on the considered component of $\left|L_{i}\right|^{G}$. The basis $B$ parameterizing such complete intersections is thus a Zariski open set in $\prod_{i} \mathbb{P}\left(H^{0}\left(X, L_{i}\right)^{\chi_{i}}\right)$. As before we denote by $\chi \rightarrow B$ the universal complete intersection.

Theorem 4.2. - Assume

(i) The variety $\chi \times_{B} \chi$ satisfies property $\mathscr{P}_{n-r}$.

(ii) The Hodge structure on $H^{n-r}\left(X_{b}, \mathbb{Q}\right)_{\text {van }}^{\chi}$ is supported on a closed algebraic subset $Y_{b} \subset X_{b}$ of codimension c. (Conjecturally, this is satisfied if the Hodge coniveau of $H^{n-r}\left(X_{b}, \mathbb{Q}\right)_{\text {van }}^{\chi}$ is $\geq c$, cf. Conjecture 1.1.)

(iii) Conjecture 1.6 holds for codimension $n-r$ cycles.

Then the cycle map $C H_{i}\left(X_{b}\right)_{\mathbb{Q}}^{\chi} \rightarrow H^{2 n-2 r-2 i}\left(X_{b}, \mathbb{Q}\right)^{\chi}$ is injective for any $b \in B$.

Remark 4.3. - In the case where $X_{b}$ are surfaces with $h^{2,0}\left(X_{b}\right)^{\chi}=0$, the assumption (ii) is automatically satisfied, by the same arguments as in the proof of Theorem 1.9. We thus get an alternative proof of the main theorem of [29], where the Bloch conjecture is proved for the general Godeaux surfaces (quotients of quintic surfaces by a free action of $\mathbb{Z} / 5 \mathbb{Z}$, or quotients of complete intersections of four quadrics in $\mathbb{P}^{6}$ by a free action of $\mathbb{Z} / 8 \mathbb{Z}$ ).

In the case of threefolds $X_{b}$ of Hodge coniveau 1, we can also conclude, using the obvious extension of Theorem 1.9 to the case of varieties with group action, that $C H_{0}\left(X_{b}\right)_{0}^{\chi}=0$ if (i) is satisfied and the generalized Hodge conjecture is satisfied by the coniveau 1 Hodge structure on $H^{3}\left(X_{b}, \mathbb{Q}\right)^{\chi}$. This way the second result of [29] (quintic hypersurfaces with involutions) and the main application of [23] (3-dimensional complete intersection in weighted projective space) are reproved : in both cases we are essentially reduced to prove the generalized Hodge conjecture for the coniveau 1 Hodge structure on their cohomology of degree 3.

Example 4.4. - Consider a Calabi-Yau hypersurface $X_{f}$ in $X=\mathbb{P}^{n}$ defined by an equation $f$ invariant by the involution $i: i^{*}\left(X_{0}, \ldots, X_{n}\right)=\left(-X_{0},-X_{1}, X_{2}, \ldots, X_{n}\right)$. Then $H^{n-1}(X)_{\text {prim }}^{-}$has coniveau 1 , since $i$ acts by Id on $H^{n-1,0}\left(X_{f}\right)$. In [29], the case of 2-dimensional quartics and 3-dimensional quintics are studied, and it is proved there that $i$ acts by Id on $C H_{0}(X)$ in both cases. One step in the 3-dimensional situation is the proof that the generalized Hodge conjecture holds for the coniveau 1 Hodge structure $H^{3}(X, \mathbb{Q})^{-}$. Having this, Theorem 4.2 gives a drastically simplified proof of this result.

EXAmple 4.5. - The following class of examples is constructed in [5]: $X=\mathbb{P}^{1} \times \mathbb{P}^{3}$, with the following group action: $G=\mathbb{Z} / 5 \mathbb{Z}$ acts on $\mathbb{P}^{1} \times \mathbb{P}^{3}$ in the following linearized way: Let $g$ be a generator of $G$ and $\zeta$ a nontrivial 5-th root of unity. Then if $x, y$ are homogeneous coordinates on $\mathbb{P}^{1}$ and $x_{0}, x_{1}, x_{2}, x_{3}$ are homogeneous coordinates on $\mathbb{P}^{3}$, we set:

$$
\begin{aligned}
g^{*} x & =x, g^{*} y=\zeta y, \\
g^{*} x_{i} & =\zeta^{i} x_{i}, i=0, \ldots, 3 .
\end{aligned}
$$

We then consider hypersurfaces $X_{f} \subset \mathbb{P}^{1} \times \mathbb{P}^{3}$ defined by an equation $f=0$ of bidegree $(3,4)$, where $f \in H^{0}\left(\mathbb{P}^{1} \times \mathbb{P}^{3}, \Theta_{\mathbb{P}^{1} \times \mathbb{P}^{3}}(3,4)\right)^{G}$.

These hypersurfaces $X_{f}$ have a few ordinary quadratic singularities. The varieties $X_{f}^{\prime}$ obtained as a desingularization of $X_{f} / G$ have $h^{3,0}\left(X_{f}^{\prime}\right)=0$ (and also $h^{i, 0}\left(X_{f}^{\prime}\right)=0$ for $i=1,2)$. For the general such variety, Theorem 4.2 tells that the generalized Hodge conjecture for $H^{3}\left(X_{f}^{\prime}, \mathbb{Q}\right.$ ) implies (and in fact is equivalent to by Theorem 1.2) the equality 
$C H_{0}\left(X_{f}^{\prime}\right)=\mathbb{Z}$. The interest in these examples comes from the fact, proved in [5], that the Hodge conjecture is not satisfied by integral Hodge classes of degree 4 on $X_{f}^{\prime}$.

\subsection{Self-products}

Let $Y$ be a smooth projective variety. We will assume for simplicity that $H^{i, 0}(Y)=0$ for $i \neq 0, m:=\operatorname{dim} Y$. (This will be the case if $Y$ is a complete intersection of ample hypersurfaces in a projective variety with trivial Chow groups.)

Lemma 4.6. - For $k>p_{g}(Y)=h^{m, 0}(Y)$, the Hodge structure of weight $k m$ on $\bigwedge^{k} H^{m}(Y, \mathbb{Q})$ has coniveau $\geq 1$. In particular, if $h^{m, 0}(Y)=1$, the Hodge structure of weight $2 m$ on $\bigwedge^{2} H^{m}(Y, \mathbb{Q})$ has Hodge coniveau $\geq 1$.

Proof. - Indeed, the $(k m, 0)$-piece of the Hodge structure on $\bigwedge^{k} H^{m}(Y, \mathbb{Q})$ is equal to $\bigwedge^{k} H^{m, 0}(Y)$, hence it is 0 for $k>h^{m, 0}(Y)$.

Conjecture 1.3 (or rather its generalization to motives) predicts the following (see below for more details):

CONJeCture 4.7. - Assume $Y$ satisfies the above assumption and has $h^{m, 0}=1$. Then, for any $z, z^{\prime} \in C H_{0}(Y)$ with $\operatorname{deg} z=\operatorname{deg} z^{\prime}=0$, one has $z \times z^{\prime}-z^{\prime} \times z=0$ in $C H_{0}(Y \times Y)$ for $m$ even and $z \times z^{\prime}+z^{\prime} \times z=0$ in $C H_{0}(Y \times Y)$ for $m$ odd.

The case $m=2$ is particularly interesting, as noticed in [30]. In this case, we indeed have :

Lemma 4.8. - Let $H, H^{p, q}$ be a weight 2 Hodge structure of $K 3$ type, namely $h^{2,0}=1$. Then the Hodge structures on $\bigwedge^{k} H$ all have niveau $\leq 2($ that is coniveau $\geq k-1)$.

Proof. - Write $H=H^{2,0} \oplus H^{1,1} \oplus H^{0,2}$. Then

$$
\bigwedge^{k} H=H^{2,0} \otimes \bigwedge^{k-1} H^{1,1} \oplus\left(\bigwedge^{k} H^{1,1} \oplus H^{2,0} \otimes H^{0,2} \otimes \bigwedge^{k-2} H^{1,1}\right) \oplus \bigwedge^{k-1} H^{1,1} \otimes H^{0,2}
$$

is the Hodge decomposition of $\bigwedge^{k} H$, whose first nonzero term is of type $(k+1, k-1)$.

When $k>\operatorname{dim} H$, we of course have that the Hodge structure on $\bigwedge^{k} H$ is trivial. Applying these observations to the case where $H=H^{2}(S, \mathbb{Q})$ where $S$ is an algebraic $K 3$ surface, we find that Conjecture 1.3 (or rather, its extension to motives) predicts the following (cf. [30]):

Conjecture 4.9. - (i) Let $S$ be an algebraic $K 3$ surface. Then for any $k \geq 2$, and $i \leq k-2$, the projector $\pi_{\text {alt }}=\sum_{\sigma \in \mathfrak{S}_{k}}(-1)^{\epsilon(\sigma)} \sigma \in C H^{2 k}\left(S^{k} \times S^{k}\right)$ composed with the Chow-Künneth projector $\pi_{2}^{\otimes k}$ (cf. [18]) acts as 0 on $C H_{i}\left(S^{k}\right)_{\mathbb{Q}}$ for $i \leq k-2$.

(ii) For $k>b_{2}(S)$, this projector is identically 0 . 
Note that (ii) above is essentially Kimura's finite dimensionality conjecture [12] and applies to any regular surface. One may wonder whether it could be attacked by the methods of the present paper for the case of quartic $K 3$ surfaces. The question would be essentially to study whether the fibered product $\chi^{2 k / B}$ of the universal such $K 3$ surface satisfies property $\mathscr{P}$. For small $k$, this can be done by the methods used in the proof of Proposition 4.11 below, but we would need to know this in the range $k \geq 44$ in order to prove the Kimura conjecture. This seems to be very hard.

The fact that this is true for small $k$ (see below) shows that Conjecture 4.9 is implied by the generalized Hodge conjecture for the self-products $S^{k}$ and the coniveau $k-1$ Hodge structures $\bigwedge^{k} H^{2}(S, \mathbb{Q}) \subset H^{2 k}\left(S^{k}, \mathbb{Q}\right)$.

Let us be a little more explicit in the case of general Calabi-Yau complete intersections and for $k=2$. Let $X_{b}$ be a smooth Calabi-Yau complete intersection of dimension $m$ in projective space $\mathbb{P}^{n}$. Let $\Delta_{b \text {,van }} \in C H^{m}\left(X_{b} \times X_{b}\right)_{\mathbb{Q}}$ be the corrected diagonal, whose action on $H^{*}\left(X_{b}, \mathbb{Q}\right)$ is the projection on $H^{m}\left(X_{b}, \mathbb{Q}\right)_{\text {van }}$. On $X_{b} \times X_{b} \times X_{b} \times X_{b}$, there is the induced $2 m$-cycle

$$
\Delta_{b, \mathrm{van}, 2}:=p_{13}^{*} \Delta_{b, \mathrm{van}} \cdot p_{24}^{*} \Delta_{b, \mathrm{van}}
$$

where $p_{i j}$ is the projection from $X_{b}^{4}$ to the product $X_{b}^{2}$ of its $i$-th and $j$-th factor. The action on $\Delta_{b, \text { van, } 2}$ seen as a self-correspondence of $X_{b}^{2}$ on $H^{*}\left(X_{b}^{2}, \mathbb{Q}\right)$ is the orthogonal projector on

$$
p_{1}^{*} H^{m}\left(X_{b}, \mathbb{Q}\right)_{\operatorname{van}} \otimes p_{2}^{*} H^{m}\left(X_{b}, \mathbb{Q}\right)_{\operatorname{van}} \subset H^{2 m}\left(X_{b} \times X_{b}, \mathbb{Q}\right) .
$$

If instead of $\Delta_{b, \mathrm{van}, 2}$, we consider

$$
\Delta_{b, \mathrm{van}, 2}^{\tau}:=p_{14}^{*} \Delta_{b, \mathrm{van}} \cdot p_{23}^{*} \Delta_{b, \mathrm{van}}
$$

then the action on $\Delta_{b, \mathrm{van}, 2}$ seen as a self-correspondence of $X_{b}^{2}$ on $H^{*}\left(X_{b}^{2}, \mathbb{Q}\right)$ is the composition of the previous projector with the permutation

$$
\tau_{*}: H^{m}\left(X_{b}, \mathbb{Q}\right)_{\text {van }} \otimes H^{m}\left(X_{b}, \mathbb{Q}\right)_{\text {van }} \rightarrow H^{m}\left(X_{b}, \mathbb{Q}\right)_{\text {van }} \otimes H^{m}\left(X_{b}, \mathbb{Q}\right)_{\text {van }}
$$

exchanging summands. Note that the inclusion

$$
H^{m}\left(X_{b}, \mathbb{Q}\right)_{\text {van }} \otimes H^{m}\left(X_{b}, \mathbb{Q}\right)_{\text {van }} \subset H^{2 m}\left(X_{b} \times X_{b}, \mathbb{Q}\right)
$$

sends the antiinvariant part on the left to the antiinvariant part under $\tau$ on the right if $m$ is even, and to the invariant part under $\tau$ on the right if $m$ is even. This is due to the fact that the cup-product on cohomology is graded commutative.

Hence we conclude that

$$
\Delta_{b, \mathrm{van}, 2}^{\sharp}:=\Delta_{b, \mathrm{van}, 2}-\Delta_{b, \mathrm{van}, 2}^{\tau}
$$

acts on $H^{*}\left(X_{b}^{2}, \mathbb{Q}\right)$ as twice the projector onto $\bigwedge^{2} H^{m}\left(X_{b} \times X_{b}, \mathbb{Q}\right)_{\text {van }}$ if $m$ is even, and that

$$
\Delta_{b, \mathrm{van}, 2}^{\mathrm{inv}}:=\Delta_{b, \mathrm{van}, 2}+\Delta_{b, \mathrm{van}, 2}^{\tau}
$$

acts on $H^{*}\left(X_{b}^{2}, \mathbb{Q}\right)$ as twice the projector onto $\bigwedge^{2} H^{m}\left(X_{b} \times X_{b}, \mathbb{Q}\right)_{\text {van }}$ if $m$ is odd.

In both cases, using Lemma 4.6, we get that this is twice the orthogonal projector associated to a sub-Hodge structure of Hodge coniveau $\geq 1$.

Restricting to the case of Calabi-Yau hypersurfaces in $\mathbb{P}^{n}$ (so $m=n-1$ ), an easy adaptation of the proof of Theorem 1.7 gives now: 
Theorem 4.10 (cf. Theorem 1.11). - Assume Conjecture 1.6 holds and the generalized Hodge conjecture holds for the coniveau 1 Hodge structure on $\bigwedge^{2} H^{n-1}\left(X_{b} \times X_{b}, \mathbb{Q}\right)_{\text {van }} \subset$ $H^{2 n-2}\left(X_{b} \times X_{b}, \mathbb{Q}\right)$, where $X_{b}$ is a general Calabi-Yau hypersurface in projective space. Then the general such $X_{b}$ has the following property:

(i) If $n-1$ is even, for any two 0 -cycles $z, z^{\prime}$ of degree 0 on $X_{b}$, we have $z \times z^{\prime}-z^{\prime} \times z=0$ in $\mathrm{CH}_{0}(\mathrm{X} \times \mathrm{X})$.

(ii) If $n-1$ is odd, for any two 0 -cycles $z, z^{\prime}$ of degree 0 on $X_{b}$, we have $z \times z^{\prime}+z^{\prime} \times z=0$ in $\mathrm{CH}_{0}(\mathrm{X} \times \mathrm{X})$.

Proof. - We just sketch the proof, as it is actually a variant of the proof of Theorem 1.7. With the same notations as in 3.9 (where $X$ will be the projective space $\mathbb{P}^{n}$ ), we claim that it suffices to show that, if $n-1$ is even, the spread-out cycles

$$
\mathscr{D}_{\mathrm{van}, 2}^{\sharp}:=\mathscr{D}_{\mathrm{van}, 2}-\mathscr{D}_{\mathrm{van}, 2}^{\tau} \in C H^{2 n-2}\left(\chi \times_{B} \chi \times_{B} \chi \times_{B} \chi\right)_{\mathbb{Q}}
$$

can be written as a sum

$$
\mathscr{D}_{\mathrm{van}, 2}^{\sharp}=Z_{1}+Z_{2} \text { in } C H^{2 m}\left(\chi_{\times_{B}} \chi \times_{B} \chi \times_{B} \chi\right)_{\mathbb{Q}},
$$

where $Z_{1}$ is supported on $\mathcal{Y} \times_{B} Y$, with $Y \varsubsetneqq \chi \times_{B} \chi$, and $Z_{2}$ is a cycle which is the restriction of cycles on various copies of $\chi \times_{B} \chi \times B \times X \times X$ (ordered adequately), via the inclusion $\chi \subset B \times X$; similarly for $n-1$ odd, with $\mathscr{D}_{\text {van }, 2}^{\sharp}$ replaced by

$$
\mathscr{D}_{\mathrm{van}, 2}^{\mathrm{inv}}:=\mathscr{D}_{\mathrm{van}, 2}+\mathscr{D}_{\mathrm{van}, 2}^{\tau} \in C H^{2 n-2}\left(\chi \times_{B} \chi \times_{B} \chi \times_{B} \chi\right)_{\mathbb{Q}} .
$$

Indeed, if we know this, restricting to a general point $b \in B$, we get that for $n-1$ even $\Delta_{\text {van }, 2}^{\sharp}:=\Delta_{\text {van }, 2}-\Delta_{\text {van }, 2}^{\tau} \in C H^{2 n-2}\left(X_{b}^{4}\right)_{\mathbb{Q}}$ can be written as a sum

$$
\Delta_{\mathrm{van}, 2}^{\sharp}=Z_{1}+Z_{2},
$$

where $Z_{1}$ is supported on $\mathscr{Y}_{b} \times \mathscr{Y}_{b}$, with $\mathscr{Y}_{b} \varsubsetneqq X_{b} \times X_{b}$, and $Z_{2}$ is a cycle which is the restriction of cycles on various copies of $X_{b} \times X_{b} \times X \times X$ (ordered adequately), via the inclusion $j_{b}: X_{b} \hookrightarrow X=\mathbb{P}^{n}$. Similarly for $n-1$ odd with $\Delta_{\text {van }, 2}^{\sharp}$ replaced by $\Delta_{\text {van }, 2}^{\text {inv }}$. We see Equation (16) as an equality of self-correspondences of $X_{b}^{2}$ and we let both sides of (16) act on $z \times z^{\prime}$, where $z, z^{\prime} \in C H_{0}\left(X_{b}\right)$ have degree 0 . On the left, we get $\Delta_{\text {van }, 2}^{\sharp}\left(z \times z^{\prime}\right)=z \times z^{\prime}-z^{\prime} \times z$. Next the cycle $Z_{1}$ being supported on $\mathscr{Y}_{b} \times \mathscr{Y}_{b}$, with $Y_{b} \varsubsetneqq X_{b} \times X_{b}$, acts trivially on $C H_{0}\left(X_{b} \times X_{b}\right)$. We thus get (for $n-1$ even), decomposing $Z_{2}$ as a sum $Z_{2}=\sum_{i} Z_{2, i \mid X_{b}^{4}}$ where $Z_{2, i} \in C H^{2 n-2}\left(X_{b} \times \cdots \times X \times \ldots X_{b}\right)$, with the factor $X$ put in $i$-th position:

$$
z \times z^{\prime}-z^{\prime} \times z=\sum_{i}\left(Z_{2, i \mid X_{b}^{4}}\right)_{*}\left(z \times z^{\prime}\right) \text { in } C H_{0}\left(X^{2}\right)_{\mathbb{Q}} .
$$

If $i=1,2,\left(Z_{2, i \mid X_{b}^{4}}\right)_{*}\left(z \times z^{\prime}\right)$ vanishes because, as $C H_{0}(X)=C H_{0}\left(\mathbb{P}^{n}\right)=\mathbb{Z}$, both cycles $z \times j_{b *} z^{\prime} \in C H_{0}\left(X_{b} \times X\right)$ and $j_{b *} z \times z^{\prime} \in C H_{0}\left(X \times X_{b}\right)$ vanish. For $i=3$, 4, we have that $\left(Z_{2, i \mid X_{b}^{4}}\right)_{*}\left(z \times z^{\prime}\right)$ belongs to $C H_{1}\left(X_{b} \times X\right)_{\mid X_{b} \times X_{b}}$ or to $C H_{1}\left(X \times X_{b}\right)_{\mid X_{b} \times X_{b}}$ with $X=\mathbb{P}^{n}$. Thus we get using the decomposition of $C H_{1}\left(X_{b} \times \mathbb{P}^{n}\right)$ as $C H_{0}\left(X_{b}\right) \otimes \mathbb{Z} h_{1} \oplus C H_{1}\left(X_{b}\right) \times \mathbb{Z} h_{0}$, where $h_{1}$ is the class of a line and $h_{0}$ is the class of a point in $\mathbb{P}^{n}$, an equality:

$$
z \times z^{\prime}-z^{\prime} \times z=w_{1} \times h_{1 \mid X_{b}}+h_{1 \mid X_{b}} \times w_{2} \text { in } C H_{0}\left(X_{b} \times X_{b}\right)_{\mathbb{Q}},
$$


with $w_{i} \in C H_{0}\left(X_{b}\right)$. Applying $p r_{1 *}$ and $p r_{2 *}$ to both sides of this equality, we finally get that $w_{1}=w_{2}=0$ in $C H_{0}\left(X_{b}\right)_{\mathbb{Q}}$. Thus we proved assuming (15) that $z \times z^{\prime}-z^{\prime} \times z=0$ in $C H_{0}\left(X_{b}^{2}\right)_{\mathbb{Q}}$ for $n-1$ even and the same proof will show that $z \times z^{\prime}+z^{\prime} \times z=0$ in $C H_{0}\left(X_{b}^{2}\right)_{\mathbb{Q}}$ for $n-1$ odd. As these cycles belong to the kernel of the Albanese map, we also conclude by Roitman's theorem [24] that these equalities in fact hold in $\mathrm{CH}_{0}\left(\mathrm{X}_{b}^{2}\right)$.

It remains to see how to get (15) from the condition that the generalized Hodge conjecture holds for the coniveau 1 Hodge structure on $\bigwedge^{2} H^{n-1}\left(X_{b}\right)_{\text {prim }}$ combined with Conjecture 1.6. As in the proof of Theorem 1.7, we find that under these two assumptions, we have an equality of cycle classes

$$
\left[\mathscr{D}_{\operatorname{van}, 2}^{\sharp}\right]=\left[\mathcal{Z}_{1}\right]+\left[\mathcal{Z}_{2}\right] \text { in } H^{4 n-4}\left(\chi \times_{B} \chi \times_{B} \chi \times_{B} \chi, \mathbb{Q}\right),
$$

where $Z_{1}$ is supported on $\mathcal{Y} \times_{B} \mathcal{Y}$, with $\mathcal{Y} \varsubsetneqq \chi \times_{B} \chi$, and $Z_{2}$ is a cycle which is the restriction of cycles on various copies of $\chi \times_{B} \chi \times B \times X \times X$.

Equation (15) follows from (18) and from the following Proposition 4.11. This finishes the proof of Theorem 1.11.

Proposition 4.11. - Assume Conjecture 1.6. Then the fourth fibered product $\chi \times_{B} \chi \times_{B}$ $\chi \times_{B} \chi$ of universal hypersurfaces of degree $\geq 3$ in $\mathbb{P}^{n}$ satisfies property $\mathscr{P}_{2 n-2}$.

Proof. - As we are interested into cycles of codimension $\leq 2 n-2$, we can restrict to the open set $\chi_{0}^{4 / B}$ defined as the complement of the small relative diagonal $\chi \subset \chi_{0}^{4 / B}$ which is of codimension $3 n-3$.

We apply the relative version of Lemma 4.12 below. This provides us with a certain blowup $\widetilde{\chi_{0}^{4 / B}}$ of the relative Fulton-MacPherson configuration space (cf. [8]). It is smooth and proper over $\chi_{0}^{4 / B}$. In order to prove the result, it suffices by Lemma 3.4 to show that $\widetilde{\chi_{0}^{4 / B}}$ satisfies property $\mathscr{P}_{2 n-2}$. By the functoriality statement in Lemma 4.12, there is a morphism from $\widehat{\chi_{0}^{4 / B}}$ to $\widehat{\left(\mathbb{P}^{n}\right)_{0}^{4}}$ and in particular to the punctual Hilbert scheme $\operatorname{Hilb}^{4}\left(\mathbb{P}^{n}\right)$, so that an element of $\widehat{\chi_{0}^{4 / B}}$ determines a 4-uple $\left(x_{1}, \ldots, x_{4}\right) \in X_{b}$ together with a subscheme $z$ of $X_{b} \subset \mathbb{P}^{n}$ of length 4 with associated cycle $x_{1}+\cdots+x_{4}$. It is an easy result that any subscheme $z$ of length 4 of $\mathbb{P}^{n}$ whose support consists of at least two points imposes independent conditions to degree $n+1$ hypersurfaces, with $n+1 \geq 3$. It follows that $\widehat{\chi_{0}^{4 / B}}$ can be realized as a Zariski open set of a projective bundle over the space $\widetilde{\left(\mathbb{P}^{n}\right)_{0}^{4}}$ constructed below. Namely, over a point $u$ in this space, giving rise to $x_{1}, \ldots, x_{4}$ together with a schematic structure $z$ with associated cycle $x_{1}+\cdots+x_{4}$, the fiber is the projective space $\mathbb{P}\left(H^{0}\left(\mathbb{P}^{n}, \searrow_{z}(n+1)\right)\right)$, and we have to take the Zariski open set in it parameterizing smooth hypersurfaces.

Lemma 3.3 says that $\widetilde{\left(\mathbb{P}^{n}\right)_{0}^{4}}$ satisfies property $\mathscr{P}_{2 n-2}$. By Lemma 3.2, it follows that the projective bundle described above over $\widetilde{\left.\mathbb{P}^{n}\right)_{0}^{4}}$ also satisfies $\mathscr{P}_{2 n-2}$. By Lemma 3.1, the Zariski open set $\widetilde{\chi_{0}^{4 / B}}$ inside it also satisfies $\mathscr{P}_{2 n-2}$.

Lemma 4.12 (cf. [15]). - Let $X$ be a smooth variety of any dimension $n$. Denote by $X_{0}^{4}$ the open set $X^{4} \backslash \Delta_{X, 4}$, where $\Delta_{X, 4} \cong X$ is the small diagonal. There is a smooth variety $\widetilde{X_{0}^{4}}$ which admits a morphism to $\operatorname{Hilb}^{4}(X)$ (so the rational map $\sigma: X_{0}^{4} \rightarrow \operatorname{Hilb}^{4}(X)$ is desingularized 
on $\widetilde{X_{0}^{4}}$ ), whose construction is functorial under immersions, and which satisfies property $\mathscr{P}_{2 n-2}$ if $X$ satisfies property $\mathscr{D}$.

Proof. - We will just describe the construction of $\widetilde{X_{0}^{4}}$ over a neighborhood in $X_{0}^{4}$ of a point of $X_{0}^{4}$ of type $(3,1)$, that is a point which corresponds to a relative 0 -cycle of the form $3 x+y$. The case of points of type $(2,2)$ (that is a point which corresponds to a 0 -cycle of the form $2 x+2 y$ ) or with a support of cardinal $\geq 3$ is easy and left to the reader. As the set of points of type $(3,1)$ and $(2,2)$ are disjoint in $X_{0}^{4}$ there is no problem to glue the local constructions.

We first blow up inside $X_{0}^{4}$ the union of the images under permutation of the diagonals of type $\Delta_{x x x y}$ parameterizing the points

$$
(x, x, x, y) \in X_{0}^{4}, x, y \in X, x \neq y .
$$

We then blow up the (disjoint union of the) proper transforms of the images under permutations of the big diagonal $\Delta_{x x y z}$ which is defined as the closure of the set of points

$$
(x, x, y, z) \in X_{0}^{4}, x, y, z \in X, x, y, z \text { distinct. }
$$

What we get at this point is nothing but the Fulton-MacPherson compactification $X(4)_{0}$ of the configuration space of 4 points, at least over the open set $X_{0}^{4}$ of $X^{4}$ (cf. [8]).

The rational map $\sigma: X(4)_{0} \rightarrow \operatorname{Hilb}^{4}(X)$ is not yet a morphism, as shown to us by Totaro. What we need to blow up is the following locus $M$ (pointed out by Totaro): over the diagonal $\Delta_{x, x, x, y}$ (or any image of it under permutation), the exceptional divisor over $\Delta_{x, x, x, y}$ is (before the second blow-up) the projective bundle $\mathbb{P}\left(p_{x}^{*} T_{X} \oplus p_{x}^{*} T_{X}\right)$ where $p_{x}$ is the first projection $\Delta_{x x x y} \cong X \times X \rightarrow X$. Let $M^{\prime} \subset \mathbb{P}\left(p_{x}^{*} T_{X} \oplus p_{x}^{*} T_{X}\right)$ be the locus of couples $\left(v_{1}, v_{2}\right)$ where $v_{1}, v_{2}$ are colinear. $M^{\prime}$ is isomorphic to $\mathbb{P}^{1} \times \mathbb{P}\left(p_{x}^{*} T_{X}\right)$. Let $M$ be the proper transform of $M^{\prime}$ under the second blow-up, that is in $X(4)_{0} . M$ is isomorphic to $M^{\prime}$.

It is explained in the letter [15] that $\sigma$ becomes well-defined on the blow-up of $X(4)_{0}$ along $M$, as a consequence of Hayman's theorem [11]. This of course concerns the case where $\operatorname{dim} X=2$. However, we are looking at $\operatorname{Hilb}^{4}(X)_{0}$, the open set of $\operatorname{Hilb}^{4}(X)$ where the support has cardinality at least 2 , and in fact are mostly concerned with the neighborhood in $\operatorname{Hilb}^{4}(X)$ of punctual subschemes of type $(3,1)$. As this is locally (in the étale or analytic topology) isomorphic to the product $X \times \operatorname{Hilb}^{3}(X)$, we are reduced to study the case of $\operatorname{Hilb}^{3}(X)$ in a neighborhood of a fat point $z$. We observe now that any length 3 subscheme $z \subset X$ is contained in a smooth surface in $X$. More precisely, if $n$ is the dimension of $X$, we choose a linear system of hypersurfaces $H_{0} \ldots, H_{n}$ in $X$ with the property that $z$ imposes 3 independent conditions to $\left\langle H_{0}, \ldots, H_{n}>\right.$, and that the locus $\Sigma_{z} \subset X$ defined by the linear subsystem $I_{z} \subset<H_{0}, \ldots, H_{n}>$ is smooth. The map

$$
\begin{aligned}
\phi: \operatorname{Hilb}^{3}(X) & \rightarrow \operatorname{Grass}(3, n+1), \\
z^{\prime} & \mapsto I_{z^{\prime}} \subset<H_{0}, \ldots, H_{n}>
\end{aligned}
$$

is then well-defined near $z$, dominant, with fibers through a point $z^{\prime} \in \operatorname{Hilb}^{3}(X)$ close to $z$ the Hilbert scheme $\operatorname{Hilb}^{3}\left(\Sigma_{z^{\prime}}\right)$ of the smooth surface $\Sigma_{z^{\prime}}$. Using this, we easily reduce the general case to the surface case.

Applying Lemmas 3.3 and 3.1 , it is easy to show that the resulting variety $\widetilde{X_{0}^{4}}$, satisfies property $\mathscr{P}_{2 n-2}$ if $X$ satisfies property $\mathscr{P}$. 


\section{REFERENCES}

[1] A. Albano, A. Collino, On the Griffiths group of the cubic sevenfold, Math. Ann. 299 (1994), 715-726.

[2] S. BLOch, Lectures on algebraic cycles, second ed., New Mathematical Monographs 16, Cambridge Univ. Press, 2010.

[3] S. Bloch, V. SRinivas, Remarks on correspondences and algebraic cycles, Amer. J. Math. 105 (1983), 1235-1253.

[4] F. CharLes, Remarks on the Lefschetz standard conjecture and hyperkähler varieties, preprint 2010, to appear in Comm. Math. Helv.

[5] J.-L. Colliot-Thélène, C. Voisin, Cohomologie non ramifiée et conjecture de Hodge entière, Duke Math. J. 161 (2012), 735-801.

[6] P. Deligne, Théorème de Lefschetz et critères de dégénérescence de suites spectrales, Publ. Math. I.H.É.S. 35 (1968), 259-278.

[7] H. Esnault, M. Levine, E. Viehweg, Chow groups of projective varieties of very small degree, Duke Math. J. 87 (1997), 29-58.

[8] W. Fulton, R. MacPherson, A compactification of configuration spaces, Ann. of Math. 139 (1994), 183-225.

[9] M. Green, P. Griffiths, Hodge-theoretic invariants for algebraic cycles, Int. Math. Res. Not. 2003 (2003), 477-510.

[10] A. Grothendieck, Hodge's general conjecture is false for trivial reasons, Topology 8 (1969), 299-303.

[11] M. Haiman, Hilbert schemes, polygraphs and the Macdonald positivity conjecture, J. Amer. Math. Soc. 14 (2001), 941-1006.

[12] S.-I. Kimura, Chow groups are finite dimensional, in some sense, Math. Ann. 331 (2005), 173-201.

[13] S. L. Kleiman, Algebraic cycles and the Weil conjectures, in Dix exposés sur la cohomologie des schémas, North-Holland, 1968, 359-386.

[14] R. Laterveer, Algebraic varieties with small Chow groups, J. Math. Kyoto Univ. 38 (1998), 673-694.

[15] M. Lehn, C. Sorger, Letter to the author, June 24th, 2011.

[16] J. D. Lewis, A generalization of Mumford's theorem. II, Illinois J. Math. 39 (1995), 288-304.

[17] D. Mumford, Rational equivalence of 0-cycles on surfaces, J. Math. Kyoto Univ. 9 (1968), 195-204.

[18] J. P. Murre, On the motive of an algebraic surface, J. reine angew. Math. 409 (1990), 190-204.

[19] M. V. NorI, Algebraic cycles and Hodge-theoretic connectivity, Invent. Math. 111 (1993), 349-373.

[20] A. Otwinowska, Remarques sur les cycles de petite dimension de certaines intersections complètes, C. R. Acad. Sci. Paris Sér. I Math. 329 (1999), 141-146.

[21] A. Otwinowska, Remarques sur les groupes de Chow des hypersurfaces de petit degré, C. R. Acad. Sci. Paris Sér. I Math. 329 (1999), 51-56.

$4^{\mathrm{e}}$ SÉRIE - TOME $46-2013$ - No 3 
[22] K. H. Paranjape, Cohomological and cycle-theoretic connectivity, Ann. of Math. 139 (1994), 641-660.

[23] C. Peters, Bloch-type conjectures and an example of a three-fold of general type, Commun. Contemp. Math. 12 (2010), 587-605.

[24] A. A. Rojtman, The torsion of the group of 0-cycles modulo rational equivalence, Ann. of Math. 111 (1980), 553-569.

[25] S. SAito, Motives and filtrations on Chow groups, Invent. Math. 125 (1996), 149-196.

[26] C. Schoen, On Hodge structures and nonrepresentability of Chow groups, Compositio Math. 88 (1993), 285-316.

[27] A. J. Sommese, Submanifolds of Abelian varieties, Math. Ann. 233 (1978), 229-256.

[28] T. Terasoma, Infinitesimal variation of Hodge structures and the weak global Torelli theorem for complete intersections, Ann. of Math. 132 (1990), 213-235.

[29] C. Voisin, Sur les zéro-cycles de certaines hypersurfaces munies d'un automorphisme, Ann. Scuola Norm. Sup. Pisa Cl. Sci. 19 (1992), 473-492.

[30] C. Voisin, Remarks on zero-cycles of self-products of varieties, in Moduli of vector bundles (Sanda, 1994; Kyoto, 1994), Lecture Notes in Pure and Appl. Math. 179, Dekker, 1996, 265-285.

[31] C. Voisin, Sur les groupes de Chow de certaines hypersurfaces, C. R. Acad. Sci. Paris Sér. I Math. 322 (1996), 73-76.

[32] C. Voisin, Hodge theory and complex algebraic geometry. I and II, Cambridge Studies in Advanced Math. 76 and 77, Cambridge Univ. Press, 2002, 2003.

[33] C. Voisin, Coniveau 2 complete intersections and effective cones, Geom. Funct. Anal. 19 (2010), 1494-1513.

[34] C. Voisin, Lectures on the Hodge and Grothendieck-Hodge conjectures, Rend. Semin. Mat. Univ. Politec. Torino 69 (2011), 149-198.

(Manuscrit reçu le 19 novembre 2011; accepté, après révision, le 13 septembre 2012.)

\footnotetext{
Claire VoIsin CNRS et École polytechnique Centre de mathématiques Laurent Schwartz 91128 Palaiseau Cédex, France E-mail: voisin@math.polytechnique.fr
} 